\title{
Convergence Theorems for Bregman $K$-Mappings and Mixed Equilibrium Problems in Reflexive Banach Spaces
}

\author{
Bashir Ali ${ }^{1}$ and M. H. Harbau, ${ }^{1,2}$ \\ ${ }^{1}$ Department of Mathematical Sciences, Bayero University, Kano, Nigeria \\ ${ }^{2}$ Department of Science and Technology Education, Bayero University, Kano, Nigeria
}

Correspondence should be addressed to Bashir Ali; bashiralik@yahoo.com

Received 19 May 2016; Accepted 25 July 2016

Academic Editor: Adrian Petrusel

Copyright (C) 2016 B. Ali and M. H. Harbau. This is an open access article distributed under the Creative Commons Attribution License, which permits unrestricted use, distribution, and reproduction in any medium, provided the original work is properly cited.

\begin{abstract}
We introduce a new mixed equilibrium problem with a relaxed monotone mapping in a reflexive Banach space and prove the existence of solution of the equilibrium problem. Using Bregman distance, we introduce the concept of Bregman $K$-mapping for a finite family of Bregman quasiasymptotically nonexpansive mappings and show the fixed point set of the Bregman $K$-mapping is the set of common fixed points of $\left\{T_{i}\right\}_{i=1}^{N}$. Using the Bregman $K$-mapping, we introduce an iterative sequence for finding a common point in the set of a common fixed points of the finite family of Bregman quasiasymptotically nonexpansive mappings and the set of solutions of some mixed equilibrium problems. Strong convergence of the iterative sequence is proved. Our results generalise and improve many recent results in the literature.
\end{abstract}

\section{Introduction}

Let $E$ be a real Banach space and let $E^{*}$ be the dual of $E$. Let $C$ be a nonempty closed and convex subset of $E$. A mapping $T$ : $C \rightarrow C$ is called nonexpansive if $\|T x-T y\| \leq\|x-y\| \forall x, y \in$ $C$. A point $x \in C$ is a fixed point of $T$ if $T x=x$. We denote by $F(T)$ the fixed point set of $T$; that is, $F(T)=\{x \in C: T x=x\}$. Let $g: C \times C \rightarrow \mathbb{R}$ be a bifunction. The equilibrium problem with respect to $g$ and $C$ in the sense of Blum and Oettli [1] is to find $z \in C$ such that

$$
g(z, y) \geq 0 \quad \forall y \in C .
$$

The set of solutions of equilibrium problem is denoted by $\mathrm{EP}(g)$; that is,

$$
\mathrm{EP}(g)=\{z \in C: g(z, y) \geq 0 \forall y \in C\} .
$$

In order to solve equilibrium problem (1), the bifunction $g$ is usually assumed to satisfy the following conditions:

(C1) $g(x, x)=0$ for all $x, y \in C$;

(C2) $g$ is monotone; that is, $g(x, y)+g(y, x) \leq 0$ for all $x, y \in C$;
(C3) for all $x, y, z \in C$, limsup $\sup _{t \rightarrow 0} g(t z+(1-t) x, y) \leq$ $g(x, y)$;

(C4) for all $x \in C, g(x, \cdot)$ is convex and lower semicontinuous.

Fang and Huang [2] introduced the concept of relaxed $\eta-\alpha$ monotone mapping for solving mixed equilibrium problems.

A mapping $A: C \rightarrow E^{*}$ is said to be relaxed $\eta-\alpha$ monotone (see also [3]) if there exist a mapping $\eta: C \times C \rightarrow E$ and a function $\alpha: E \rightarrow \mathbb{R}$ with $\alpha(t z)=t^{p} \alpha(z)$ for all $t>0$ and $z \in E$, where $p>1$ such that

$$
\langle A x-A y, \eta(x, y)\rangle \geq \alpha(x-y) \quad \forall x, y \in C .
$$

Particularly if $\eta(x, y)=x-y \forall x, y \in C$ and $\alpha(z)=k\|z\|^{p}$, where $p>1$ and $k>1$ are two constants, then $A$ is called $p$ monotone; see $[4,5]$.

Fang and Huang [2] proved that under some suitable assumptions, the following variational inequality is solvable: find $z \in C$ such that

$$
\langle A z, \eta(y, z)\rangle+\psi(y)-\psi(z) \geq 0 \quad \forall y \in C,
$$


where $\psi$ is a function from $C$ to $\mathbb{R} \cup\{\infty\}$. They also proved that the following inequality is equivalent to variational inequality (4): find $z \in C$ such that

$$
\langle A z, \eta(y, z)\rangle+\psi(y)-\psi(z) \geq \alpha(y-z) \quad \forall y \in C .
$$

The mixed equilibrium problem (see $[6,7]$ ) is to find $z \in C$ such that

$$
g(z, y)+\langle A z, \eta(y, z)\rangle+\psi(y)-\psi(z) \geq 0 \quad \forall y \in C .
$$

We denote the set of solutions of mixed equilibrium problem (6) by $\operatorname{EP}(g, A)$. It is easily seen that if $g(z, y)=0 \forall z, y \in C$, then mixed equilibrium problem (6) reduces to variational inequality (4). In the case of $A=0$ and $\psi=0$, then, $\operatorname{EP}(g, A)$ coincides with $\operatorname{EP}(g)$.

Equilibrium problems and mixed equilibrium problems have been used as tools for solving problems arising from linear and nonlinear programming, optimization problems, variational inequalities, fixed point problems, and also problems in physics, economics, engineering, and so forth (see, e.g., $[1,6,8-15]$ and the references therein).

Let $J: E \rightarrow 2^{E^{*}}$ be the normalised duality mapping defined by

$$
J(x)=\left\{f^{*} \in E^{*}:\left\langle x, f^{*}\right\rangle=\|x\|^{2}=\left\|f^{*}\right\|^{2}\right\},
$$

where $\langle\cdot, \cdot\rangle$ denotes the generalised duality pairing. It is well known that if $E$ is smooth, strictly convex, and reflexive, then $J$ is single-valued, one-to-one, and onto.

Let $f: E \rightarrow(-\infty,+\infty]$ be a convex function. We denote by dom $f$ the domain of $f$; that is, $\operatorname{dom} f=\{x \in E: f(x)<$ $+\infty$. The function $f$ is said to be coercive if $\lim _{\|x\| \rightarrow \infty} f(x)=$ $+\infty$. $f$ is said to be strongly coercive if $\lim _{\|x\| \rightarrow \infty}(f(x) /\|x\|)=$ $+\infty$. The Fenchel conjugate of $f$ is the function $f^{*}: E^{*} \rightarrow$ $(-\infty,+\infty]$ defined by

$$
f^{*}\left(x^{*}\right)=\sup \left\{\left\langle x^{*}, x\right\rangle-f(x): x \in E\right\} .
$$

The subdifferential of $f$ is a mapping $\partial f: E \rightarrow E^{*}$ defined by

$$
\begin{aligned}
& \partial f(x) \\
&=\left\{x^{*} \in E^{*}: f(y) \geq f(x)+\left\langle x^{*}, y-x\right\rangle\right.\forall y \in E\} \\
& \forall x \in E .
\end{aligned}
$$

It is well known that (see [16]) $x^{*} \in \partial f(x)$ if and only if $f(x)+f^{*}\left(x^{*}\right)=\left\langle x^{*}, x\right\rangle$ for all $x \in E$. It is also known that if $f: E \rightarrow(-\infty,+\infty]$ is a proper, convex, and lower semicontinuous function, then $f^{*}: E^{*} \rightarrow(-\infty,+\infty]$ is a proper, convex, and weak ${ }^{*}$ lower semicontinuous function; see, for example, [17].

For any convex function $f: E \rightarrow(-\infty,+\infty]$, let $x \in$ int dom $f$ and $y \in E$. The right-hand derivative of $f$ at $x$ in the direction $y$ is defined by

$$
f^{\circ}(x, y)=\lim _{t \rightarrow 0} \frac{f(x+t y)-f(x)}{t} .
$$

The function $f$ is said to be Gâteaux differentiable at $x$ if $\lim _{t \rightarrow 0}((f(x+t y)-f(x)) / t)$ exists for any $y \in E$. In this case $f^{\circ}(x, y)$ coincides with $\nabla f(x)$, the value of the gradient $\nabla f$ of $f$ at $x . f$ is said to be Gâteaux differentiable if it is Gâteaux differentiable at each $x \in \operatorname{int} \operatorname{dom} f$. If the limit in (10) is attained uniformly in $\|y\|=1$, then $f$ is said to be Fréchet differentiable at $x . f$ is said to be uniformly Fréchet differentiable on a subset $C$ of $E$ if the limit in (10) is attained uniformly for every $x \in C$ and $\|y\|=1$. We know that if $f$ is uniformly Fréchet differentiable on bounded subset of $E$, then $f$ is uniformly continuous on bounded set of $E$ (see, e.g., [18]).

The function $f$ is said to be essentially smooth if $\partial f$ is both bounded and single-valued on its domain. It is called essentially strictly convex if $(\partial f)^{-1}$ is locally bounded on its domain and $f$ is strictly convex on every convex subset of $\operatorname{dom} \partial f . f$ is said to be a Legendre function if it is both essentially smooth and essentially strictly convex. When the subdifferential of $f$ is single-valued, it coincides with the gradient; that is, $\partial f=\nabla f$; see, for example, [19].

For a Legendre function $f$, the following properties are well known:

(i) $f$ is essentially smooth if and only if $f^{*}$ is essentially strictly convex; see [16];

(ii) $(\partial f)^{-1}=\partial f^{*}$; see $[20]$;

(iii) $f$ is Legendre if and only if $f^{*}$ is Legendre function; see [16];

(iv) if $f$ is Legendre function, then $\nabla f$ is bijection satisfying $\nabla f=\left(\nabla f^{*}\right)^{-1}, \operatorname{ran} \nabla f=\operatorname{dom} \nabla f^{*}=\operatorname{int} \operatorname{dom} f^{*}$, and $\operatorname{ran} \nabla f^{*}=\operatorname{dom} \nabla f=\operatorname{int} \operatorname{dom} f$; see [16].

If $E$ is smooth and strictly convex, the function $f(x)=$ $(1 / p)\|x\|^{p}, 1<p<\infty$, is Legendre function; see, for example, [21]. In this case $\nabla f=J_{p}, 1<p<\infty$. In particular if $E=H$ is a Hilbert space we have $\nabla f=I$, the identity mapping.

Let $f: E \rightarrow \mathbb{R}$ be a convex and Gâteaux differentiable function. The function $D_{f}: E \times E \rightarrow \mathbb{R}$ defined by

$$
D_{f}(x, y)=f(x)-f(y)-\langle\nabla f(y), x-y\rangle
$$

$$
\forall x, y \in E
$$

is called Bregman distance corresponding to $f$; see $[22,23]$. It follows from the strict convexity of $f$ that $D_{f}(x, y) \geq$ $0 \forall x, y \in E$ and $D_{f}(x, y)=0$ if and only if $x=y$; see [24].

Bregman projection with respect to $f$ of $x \in E$ onto the nonempty closed convex subset $C$ of $E$ is the unique vector $P_{C}^{f}(x) \in C$ satisfying

$$
D_{f}\left(P_{C}^{f}(x), x\right)=\inf \left\{D_{f}(y, x): y \in C\right\} .
$$

Remark 1. If $E$ is smooth and strictly convex Banach space and $f(x)=\|x\|^{2} \forall x \in E$, then we have $\nabla f(x)=2 J(x) \forall x \in E$ and hence $D_{f}(x, y)=\|x\|^{2}-2\langle x, J y\rangle+\|y\|^{2}=\phi(x, y) \forall x, y \in$ $E$ which is the Lyapunov function introduced by Alber [25] and the Bregman projection $P_{C}^{f}(x)$ reduces to the generalised projection $\Pi_{C}(x)$ which is defined by

$$
\phi\left(\Pi_{C}(x), x\right)=\inf \{\phi(y, x): y \in C\} .
$$


If $E=H$, a Hilbert space, then the Bregman projection $P_{C}^{f}(x)$ reduces to the metric projection $P_{C}(x)$ of $H$ onto $C$.

Observing (11), we have

$$
\begin{aligned}
& D_{f}(z, x)-D_{f}(z, y)= D_{f}(y, x) \\
&+\langle\nabla f(y)-\nabla f(x), z-y\rangle \\
& \forall x, y, z \in E,
\end{aligned}
$$

which is called the three-point identity.

Let $f: E \rightarrow(-\infty,+\infty]$ be a convex, Legendre, and Gâteaux differentiable function. Following $[23,25]$ we make use of the function $V_{f}: E \times E^{*} \rightarrow[0,+\infty)$ associated with $f$ defined by

$$
\begin{aligned}
& V_{f}\left(x, x^{*}\right)=f(x)-\left\langle x, x^{*}\right\rangle+f^{*}\left(x^{*}\right) \\
& \forall x \in E, x^{*} \in E^{*} .
\end{aligned}
$$

Then $V_{f}$ is nonnegative and $V_{f}\left(x, x^{*}\right)=D_{f}\left(x, \nabla f^{*}\left(x^{*}\right)\right)$ $\forall x \in E$ and $x^{*} \in E^{*}$. Also from definition (15), it is obvious that $D_{f}(x, y)=V_{f}(x, \nabla f(y))$ and $V_{f}$ is convex in the second variable. Therefore for $t \in(0,1)$ and $x, y \in E$, we have

$$
\begin{gathered}
D_{f}\left(z, \nabla f^{*}(t \nabla f(x)+(1-t) \nabla f(y))\right) \\
\leq t D_{f}(z, x)+(1-t) D_{f}(z, y) .
\end{gathered}
$$

Moreover by subdifferential inequality [26], we have

$$
\begin{aligned}
V_{f}\left(x, x^{*}\right)+\left\langle y^{*}, \nabla f^{*}\left(x^{*}\right)-x\right\rangle \leq & V_{f}\left(x, x^{*}+y^{*}\right) \\
& \forall x \in E, x^{*} \in E^{*} .
\end{aligned}
$$

Recall that a mapping $T: C \rightarrow C$ is said to be $\phi$-quasi nonexpansive if $F(T) \neq \emptyset$ and $\phi(p, T x) \leq \phi(p, x) \forall x \in C$ and $p \in F(T) . T$ is $\phi$-quasiasymptotically nonexpansive if $F(T) \neq$ $\emptyset$ and there exists a real sequence $\left\{v_{n}\right\} \subset[0, \infty)$ such that $v_{n} \rightarrow 0$ as $n \rightarrow \infty$ and $\phi\left(p, T^{n} x\right) \leq\left(1+v_{n}\right) \phi(p, x) \forall x \in C$ and $p \in F(T)$. T is called Bregman quasi nonexpansive if $F(T) \neq \emptyset$ and $D_{f}(p, T x) \leq D_{f}(p, x) \forall x \in C$ and $p \in F(T)$. $T$ is Bregman quasiasymptotically nonexpansive if $F(T) \neq \emptyset$ and there exists a real sequence $\left\{v_{n}\right\} \subset[0, \infty)$ such that $v_{n} \rightarrow 0$ as $n \rightarrow \infty$ and $D_{f}\left(p, T^{n} x\right) \leq\left(1+v_{n}\right) D_{f}(p, x) \forall x \in C$ and $p \in F(T) . T$ is said to be closed if for any sequence $\left\{x_{n}\right\} \subset C$ with $x_{n} \rightarrow x$ and $T x_{n} \rightarrow y, T x=y$.

It is worth mentioning that several iterative schemes have been constructed and proposed for finding points which solve fixed point problems and mixed equilibrium problems with relaxed monotone mappings in various settings. In 2010 Wang et al. [7] introduced the following iterative scheme for finding a common element of the set of solutions of a mixed equilibrium problem with relaxed monotone mapping and the set of fixed points of nonexpansive mappings in Hilbert spaces:

$$
\begin{aligned}
& x_{1} \in C \quad \text { chosen arbitrarily, } \\
& \Phi\left(u_{n}, y\right)+\left\langle T u_{n}, \eta\left(y, u_{n}\right)\right\rangle+\frac{1}{\lambda_{n}}\left\langle y-u_{n}, u_{n}-x_{n}\right\rangle \\
& \quad \geq 0 \quad \forall y \in C, \\
& y_{n}=\alpha_{n} x_{n}+\left(1-\alpha_{n}\right) \beta_{n} S x_{n}+\left(1-\alpha_{n}\right)\left(1-\beta_{n}\right) u_{n}, \\
& C_{n}=\left\{z \in C:\left\|y_{n}-z\right\| \leq\left\|x_{n}-z\right\|\right\}, \\
& D_{n}=\bigcap_{j=1}^{n} C_{j}, \\
& x_{n+1}=P_{D_{n}} x_{1}, \quad n \geq 1,
\end{aligned}
$$

where $T$ is a relaxed $\eta-\alpha$ monotone mapping and $S: C \rightarrow C$ is a nonexpansive mapping. Under some mild conditions on the three control sequences $\left\{\alpha_{n}\right\},\left\{\beta_{n}\right\}$, and $\left\{\lambda_{n}\right\}$, they obtained strong convergence of scheme (18) to common solution of mixed equilibrium problems and fixed point of nonexpansive mapping.

Recently, Chen et al. [27] introduced a new mixed equilibrium problem with the relaxed monotone mapping in uniformly convex and uniformly smooth Banach spaces and proved the existence of solutions of the mixed equilibrium problem. They also proposed the following iterative scheme to find the common element of the set of solutions of the mixed equilibrium problem and the set of fixed points of a quasi- $\phi$-nonexpansive mapping:

$$
\begin{aligned}
& x_{1}=x \in C \quad \text { chosen arbitrarily, } \\
& y_{n}=J^{-1}\left(\alpha_{n} J x_{n}+\left(1-\alpha_{n}\right) J S x_{n}\right), \\
& u_{n} \in C \quad \text { such that } \\
& \theta\left(u_{n}, y\right)+\left\langle A u_{n}, \eta\left(y, u_{n}\right)\right\rangle+f(y)-f\left(u_{n}\right) \\
& \quad+\frac{1}{r_{n}}\left\langle y-u_{n}, J u_{n}-J y_{n}\right\rangle \geq 0 \quad \forall y \in C, \\
& C_{n}=\left\{z \in C: \phi\left(z, u_{n}\right) \leq \phi\left(z, x_{n}\right)\right\}, \\
& D_{n}=\bigcap_{j=1}^{n} C_{j}, \\
& x_{n+1}=\prod_{D_{n}} x, \quad n \geq 1,
\end{aligned}
$$

where $S$ is a quasi- $\phi$-nonexpansive mapping from $C$ into itself and $J$ is the normalised duality mapping. Under some assumptions on the parameter sequences $\left\{\alpha_{n}\right\}$ and $\left\{r_{n}\right\}$, they obtained strong convergence of scheme (19) to common solution of mixed equilibrium problems and fixed point of nonexpansive mapping.

Motivated and inspired by the above results, in this paper we introduce and prove the existence of solutions of the mixed equilibrium problem with relaxed monotone 
mapping in reflexive Banach spaces. Using Bregman distance, we introduce the concept of Bregman $K$-mapping of a finite family of Bregman quasiasymptotically nonexpansive mappings and propose an iterative sequence for finding a common element of the set of fixed points of a finite family of Bregman quasiasymptotically nonexpansive mappings and the set of solutions of mixed equilibrium problem.

\section{Preliminaries}

Let $f: E \rightarrow(-\infty,+\infty]$ be a convex and Gâteaux differentiable function. The modulus of total convexity of $f$ at $x \in \operatorname{int} \operatorname{dom} f$ is the function $v_{f}(x, \cdot):[0,+\infty) \rightarrow[0,+\infty)$ defined by

$$
v_{f}(x, t)=\inf \left\{D_{f}(y, x): y \in \operatorname{dom} f,\|y-x\|=t\right\}
$$

The function $f$ is totally convex at $x$ if $v_{f}(x, t)>0$ for all $t>0$ and $f$ is totally convex if it is totally convex at each point $x \in \operatorname{dom} f$. Let $B$ be a bounded subset of $E$. For $t>0$, define a functional on $B, v_{f}:$ int $\operatorname{dom} f \times[0,+\infty) \rightarrow[0,+\infty)$ defined by

$$
v_{f}(B, t)=\inf \left\{v_{f}(x, t): x \in B \cup \operatorname{dom} f\right\} .
$$

$f$ is totally convex on bounded set $B$ if $v_{f}(B, t)>0$ for any bounded subset $D$ of $E$ and $t>0$, where $v_{f}(\cdot, t)$ is the total convexity of the function $f$ on the set $B$.

Let $B_{r}=\{z \in E:\|z\| \leq r\}$, for all $r>0$ and $S_{E}=\{x \in E$ : $\|x\|=1\}$. The function $f$ is bounded if $f\left(B_{r}\right)$ is bounded for all $r>0$ and $f$ is uniformly convex on bounded subsets of $E$ [28] if the function $\rho_{r}:[0,+\infty) \rightarrow[0,+\infty)$ defined by

$$
\begin{aligned}
& \rho_{r}(t) \\
& =\inf _{x, y \in B_{r},\|x-y\|=t, \alpha \in(0,1)} \frac{\alpha f(x)+(1-\alpha) f(y)-f(\alpha x+(1-\alpha) y)}{\alpha(1-\alpha)}
\end{aligned}
$$

satisfies

$$
\rho_{r}(t)>0 \quad \forall r, t>0,
$$

where $\rho_{r}$ is called the gauge of uniform convexity of $f$.

The gauge of uniform smoothness of $f$ is the function $\sigma_{r}$ : $[0,+\infty) \rightarrow[0,+\infty)$ defined by

$$
\sigma_{r}(t)=\sup _{x \in B_{r}, y \in S_{E}, \alpha \in(0,1)} \frac{\alpha f(x+(1-\alpha) t y)+(1-\alpha) f(x-t \alpha y)-f(x)}{\alpha(1-\alpha)} .
$$

The function $f$ is said to be uniformly smooth on $E$ if $\sigma_{r}(t)>$ $0 \forall r, t>0$. We know that from $[28,29] f$ is totally convex on bounded sets if and only if $f$ is uniformly convex on bounded sets.

Definition 2 (see [2]). Let $E$ be a Banach space with the dual $E^{*}$ and $C$ be a nonempty closed convex subset of $E$. Let $A$ : $C \rightarrow E^{*}$ and $\eta: C \times C \rightarrow E$ be two mappings. Then $A: C \rightarrow$ $E^{*}$ is said to be $\eta$-hemicontinuous if for any fixed $x, y \in C$, the function $h:[0,1] \rightarrow \mathbb{R}$ defined by $h(t)=\langle A((1-t) x+$ ty), $\eta(x, y)\rangle$ is continuous at $0^{+}$.

Definition 3 (see [30]). Let $E$ be a Banach space with the dual $E^{*}$ and $C$ be a nonempty subset of $E$. A mapping $H: C \rightarrow 2^{E}$ is called a KKM mapping if for any finite set $B \subset C$ one has $\operatorname{co} B \subset \bigcup_{x \in B} H(x)$.

Remark 4. Observe that from Definition 3, if $H$ is a KKM mapping and $W: C \rightarrow 2^{E}$ such that $H(x) \subset W(x)$ for all $x \in C$, then $W$ is a KKM mapping.

In the sequel we will need the following lemmas.

Lemma 5 (see [31]). Let $M$ be a nonempty subset of a Hausdorff topological vector space $X$ and let $H: M \rightarrow 2^{X}$ be a KKM mapping. If $H(x)$ is closed in $X$ for all $x \in M$ and compact for some $x \in M$, then $\bigcap_{x \in M} H(x) \neq \emptyset$.
Lemma 6 (see [32]). If $x \in \operatorname{dom} f$, then the following statements are equivalent:

(i) the function $f$ is totally convex at $x$;

(ii) for any sequence $\left\{y_{n}\right\} \subset \operatorname{dom} f$

$$
\lim _{n \rightarrow \infty} D_{f}\left(y_{n}, x\right)=0 \quad \text { implies } \lim _{n \rightarrow \infty}\left\|y_{n}-x\right\|=0
$$

Recall that a function $f: E \rightarrow(-\infty,+\infty]$ is called sequentially consistent [29] if for any two sequences $\left\{x_{n}\right\}$ and $\left\{y_{n}\right\}$ in $E$ such that $\left\{x_{n}\right\}$ is bounded,

$$
\lim _{n \rightarrow \infty} D_{f}\left(y_{n}, x_{n}\right)=0 \text { implies } \lim _{n \rightarrow \infty}\left\|y_{n}-x_{n}\right\|=0
$$

Lemma 7 (see [24]). The function $f: E \rightarrow(-\infty,+\infty]$ is totally convex on bounded sets if and only if $f$ is sequentially consistent.

Lemma 8 (see [26]). Let $f: E \rightarrow(-\infty,+\infty]$ be a Legendre function such that $\nabla f^{*}$ is bounded on bounded subsets of $E^{*}$. Let $x \in E$. If the sequence $\left\{D_{f}\left(x, x_{n}\right)\right\}$ is bounded, then the sequence $\left\{x_{n}\right\}$ is bounded. 
Lemma 9 (see [33]). Let $r>0$ be a constant and let $f: E \rightarrow \mathbb{R}$ be a uniformly convex function on bounded subsets of $E$. Then for any $x, y \in B_{r}$ and $\alpha \in(0,1)$,

$$
\begin{aligned}
f(\alpha x+(1-\alpha) y) \leq & \alpha f(x)+(1-\alpha) f(y) \\
& -\alpha(1-\alpha) \rho_{r}(\|x-y\|),
\end{aligned}
$$

where $\rho_{r}$ is the gauge of the uniform convexity of $f$.

Lemma 10 (see [34]). Let $f: E \rightarrow(-\infty,+\infty]$ be a uniformly Fréchet differentiable function and bounded on bounded subsets of $E$. Then $\nabla f$ is uniformly continuous on bounded subsets of $E$ from the strong topology of $E$ to strong topology of $E^{*}$.

Lemma 11 (see [28]). Let $f: E \rightarrow(-\infty,+\infty]$ be a strongly coercive function. If $\nabla f$ is uniformly continuous on bounded subsets of $E$, then $f^{*}: E^{*} \rightarrow(-\infty,+\infty]$ is uniformly convex on bounded subsets of $E^{*}$.

Lemma 12 (see [28]). Let $f: E \rightarrow(-\infty,+\infty]$ be a convex function which is bounded on bounded subsets of $E$. Then the following assertions are equivalent:

(i) $f$ is strongly coercive and uniformly convex on bounded subsets of $E$;

(ii) $f^{*}$ is Fréchet differentiable and $\nabla f^{*}$ is uniformly normto-norm continuous on bounded subsets of $\operatorname{dom} f^{*}=$ $E^{*}$.

Lemma 13 (see [29]). Let $C$ be a nonempty closed and convex subset of $E$. Let $f: E \rightarrow \mathbb{R}$ be a Gâteaux differentiable and totally convex function. Let $x \in E$. Then

(i) $z=P_{C}^{f}(x)$ if and only if $\langle\nabla f(x)-\nabla f(y), y-z\rangle \leq 0$ for all $y \in C$;

(ii) $D_{f}\left(y, P_{C}^{f}(x)\right)+D_{f}\left(P_{C}^{f}(x), x\right) \leq D_{f}(y, x)$ for all $y \in C$.

Lemma 14 (see [35]). Let $E$ be a reflexive Banach space and $C$ be a nonempty closed and convex subset of $E$. Let $f: E \rightarrow$ $(-\infty,+\infty]$ be a Legendre function which is bounded, uniformly Fréchet differentiable, and totally convex which is bounded on bounded subsets of E. Let T:C $\rightarrow C$ be a closed and Bregman quasiasymptotically nonexpansive mapping. If $F(T) \neq \emptyset$, then it is closed and convex.

Lemma 15. Let $E$ be a reflexive Banach space and $C$ be a nonempty closed and convex subset of $E$. Let $f: E \rightarrow$ $(-\infty,+\infty]$ be a Legendre, uniformly Fréchet differentiable, strongly coercive, and totally convex function on bounded subsets of $E$. Then the Bregman projection $P_{C}^{f}: E \rightarrow C$ is continuous.

Proof. Let $\left\{x_{n}\right\}$ be a sequence in $E$ such that $x_{n} \rightarrow x$ as $n \rightarrow$ $\infty$. Let $x_{n}^{\prime}=P_{C}^{f} x_{n}$ and $x^{\prime}=P_{C}^{f} x$. By Lemma 13(ii), we have

$$
D_{f}\left(y, x_{n}^{\prime}\right)+D_{f}\left(x_{n}^{\prime}, x_{n}\right) \leq D_{f}\left(y, x_{n}\right), \quad \forall y \in C .
$$

From inequality (28), we have

$$
D_{f}\left(y, x_{n}^{\prime}\right) \leq D_{f}\left(y, x_{n}\right), \quad \forall y \in C .
$$

Since $\left\{D_{f}\left(y, x_{n}\right)\right\}$ converges, it is bounded and using the above inequality it follows that $D_{f}\left(y, x_{n}^{\prime}\right)$ is bounded. The function $f$ is strongly coercive and totally convex which is bounded on bounded subsets of $E$; therefore in view of Lemma $12 \nabla f^{*}$ is uniformly norm-to-norm continuous on bounded subsets of $\operatorname{dom} f^{*}=E^{*}$ and consequently $\nabla f^{*}$ is bounded. Hence by Lemma 8 we obtain that $\left\{x_{n}^{\prime}\right\}$ is bounded.

Since $x^{\prime}=P_{C}^{f} x$ we have $D_{f}\left(x^{\prime}, x\right) \leq D_{f}\left(x_{n}^{\prime}, x\right)$. Therefore

$$
\begin{aligned}
& D_{f}\left(x_{n}^{\prime}, x_{n}\right)-D_{f}\left(x^{\prime}, x\right) \geq D_{f}\left(x_{n}^{\prime}, x_{n}\right)-D_{f}\left(x_{n}^{\prime}, x\right) \\
& =f(x)-f\left(x_{n}\right)+\left\langle\nabla f\left(x_{n}\right)-\nabla f(x), x_{n}-x_{n}^{\prime}\right\rangle \\
& \quad+\left\langle\nabla f(x), x_{n}-x\right\rangle .
\end{aligned}
$$

Since $f$ is uniformly Fréchet differentiable on bounded subsets of $E$, it follows that $f$ is uniformly continuous on bounded subsets of $E$ (see, e.g., [18]). Thus, taking lim inf as $n \rightarrow \infty$ of both sides of the above inequality, we obtain

$$
\liminf _{n \rightarrow \infty} D_{f}\left(x_{n}^{\prime}, x_{n}\right)-D_{f}\left(x^{\prime}, x\right) \geq 0 .
$$

Now let $\epsilon>0$. Using (28), we have

$$
\begin{aligned}
D_{f}\left(x^{\prime}, x_{n}^{\prime}\right) \leq & D_{f}\left(x^{\prime}, x_{n}\right)-D_{f}\left(x_{n}^{\prime}, x_{n}\right) \\
\leq & f(x)-f\left(x_{n}\right) \\
& +\left\langle\nabla f(x)-\nabla f\left(x_{n}\right), x^{\prime}-x\right\rangle \\
& +\left\langle\nabla f\left(x_{n}\right), x_{n}-x\right\rangle+\epsilon \quad \forall n \geq N_{0},
\end{aligned}
$$

where $N_{0}$ is some natural number. As $n \rightarrow \infty$ and $\epsilon \rightarrow 0$, we obtain

$$
D_{f}\left(x^{\prime}, x_{n}^{\prime}\right) \longrightarrow 0
$$

By total convexity of $f$, we get $x_{n}^{\prime} \rightarrow x^{\prime}$ as $n \rightarrow \infty$. This completes the proof.

\section{Main Results}

Lemma 16. Let $C$ be a nonempty, closed, and convex subset of a reflexive Banach space $E$ with the dual $E^{*}$. Let $f: E \rightarrow$ $(-\infty,+\infty]$ be a convex and Gâteaux differentiable function. Let $A: C \rightarrow E^{*}$ be $\eta$-hemicontinuous and relaxed $\eta-\alpha$ monotone mapping and $g: C \times C \rightarrow \mathbb{R}$ be a bifunction satisfying $(\mathrm{C} 1)$ and (C4). Let $\psi: C \rightarrow \mathbb{R}$ be proper, convex, and lower semicontinuous. For $r>0$ and $x \in E$, suppose the following conditions hold:

(i) $\eta(z, z)=0 \forall z \in C$;

(ii) $\langle A v, \eta(\cdot, u)\rangle$ is convex for fixed $u, v \in C$.

Then problems (34) and (35) are equivalent. 
Find $z \in C$ such that

$$
\begin{aligned}
& g(z, y)+\langle A z, \eta(y, z)\rangle+\psi(y)-\psi(z) \\
& +\frac{1}{r}\langle\nabla f(z)-\nabla f(x), y-z\rangle \geq 0 \quad \forall y \in C .
\end{aligned}
$$

Find $z \in C$ such that

$$
\begin{aligned}
& g(z, y)+\langle A y, \eta(y, z)\rangle+\psi(y)-\psi(z) \\
& \quad+\frac{1}{r}\langle\nabla f(z)-\nabla f(x), y-z\rangle \geq \alpha(y-z)
\end{aligned}
$$

$\forall y \in C$.

Proof. Suppose (34) holds. Let $z \in C$ be a solution of (34); then

$$
\begin{aligned}
& g(z, y)+\langle A z, \eta(y, z)\rangle+\psi(y)-\psi(z) \\
& +\frac{1}{r}\langle\nabla f(z)-\nabla f(x), y-z\rangle \geq 0 \quad \forall y \in C .
\end{aligned}
$$

Since $A$ is relaxed $\eta-\alpha$ monotone, we obtain

$$
\begin{aligned}
& g(z, y)+\langle A y, \eta(y, z)\rangle+\psi(y)-\psi(z) \\
& +\frac{1}{r}\langle\nabla f(z)-\nabla f(x), y-z\rangle \geq g(z, y) \\
& +\langle A z, \eta(y, z)\rangle+\psi(y)-\psi(z) \\
& +\frac{1}{r}\langle\nabla f(z)-\nabla f(x), y-z\rangle+\alpha(y-z)
\end{aligned}
$$

$\forall y \in C$

$$
\geq \alpha(y-z) \quad \forall y \in C
$$

This shows that $z \in C$ is a solution of (35).

Conversely, suppose (35) holds; that is, $z \in C$ is a solution of (35). Let $y \in C$ such that $\psi(y)<\infty$; then $\psi(z)<\infty$. Let $y_{t}=(1-t) z+t y, t \in(0,1)$. Since $z \in C$ is a solution of (35), we have

$$
\begin{aligned}
& g\left(z, y_{t}\right)+\left\langle A y_{t}, \eta\left(y_{t}, z\right)\right\rangle+\psi\left(y_{t}\right)-\psi(z) \\
& \quad+\frac{1}{r}\left\langle\nabla f(z)-\nabla f(x), y_{t}-z\right\rangle \geq \alpha\left(y_{t}-z\right) \\
& \quad=\alpha(t(y-z)) \geq t^{p} \alpha(y-z) .
\end{aligned}
$$

Hence by (C1), (C4), (i), and (ii), we obtain

$$
\begin{aligned}
t^{p} \alpha( & y-z) \\
\leq & g(z,(1-t) z+t y) \\
& +\left\langle A y_{t}, \eta((1-t) z+t y), z\right\rangle \\
& +\psi((1-t) z+t y)-\psi(z) \\
& +\frac{1}{r}\langle\nabla f(z)-\nabla f(x),((1-t) z+t y)-z\rangle \\
\leq & (1-t) g(z, z)+\operatorname{tg}(z, y) \\
& +(1-t)\left\langle A y_{t}, \eta(z, z)\right\rangle+t\left\langle A y_{t}, \eta(y, z)\right\rangle \\
& +t \psi(y)-t \psi(z) \\
& +t\left(\frac{1}{r}\langle\nabla f(z)-\nabla f(x), y-z\rangle\right) .
\end{aligned}
$$

Thus,

$$
\begin{aligned}
t^{p-1} \alpha(y-z) \leq & g(z, y)+\left\langle A y_{t}, \eta(y, z)\right\rangle+\psi(y) \\
& -\psi(z) \\
& +\frac{1}{r}\langle\nabla f(z)-\nabla f(x), y-z\rangle \\
= & g(z, y) \\
& +\langle A((1-t) z+t y), \eta(y, z)\rangle \\
& +\psi(y)-\psi(z) \\
& +\frac{1}{r}\langle\nabla f(z)-\nabla f(x), y-z\rangle .
\end{aligned}
$$

Since $A$ is $\eta$-hemicontinuous and $p>1$, by allowing $t \rightarrow 0^{+}$ we obtain

$$
\begin{aligned}
0 \leq & g(z, y)+\langle A z, \eta(y, z)\rangle+\psi(y)-\psi(z) \\
& +\frac{1}{r}\langle\nabla f(z)-\nabla f(x), y-z\rangle .
\end{aligned}
$$

This shows that $z \in C$ is a solution of (34)

Lemma 17. Let $E$ be a reflexive Banach space with the dual $E^{*}$ and let $C$ be a nonempty closed, convex, and bounded subset of $E$. Let $f: E \rightarrow(-\infty,+\infty]$ be a Legendre function and uniformly Fréchet differentiable on bounded subsets of E. Let $A: C \rightarrow E^{*}$ be $\eta$-hemicontinuous and relaxed $\eta-\alpha$ monotone mapping and $g: C \times C \rightarrow \mathbb{R}$ be a bifunction satisfying $(C 1)$ and (C4). Let $\psi: C \rightarrow \mathbb{R}$ be proper, convex lower semicontinuous. For $r>0$ and $x \in E$, suppose

(i) $\eta(x, x)=0$ for all $x \in C$,

(ii) $\eta(z, y)+\eta(y, z)=0 \forall z, y \in C$,

(iii) $\langle A u, \eta(\cdot, v)\rangle$ is convex and lower semicontinuous for fixed $u, v \in C$,

(iv) $\alpha: E \rightarrow \mathbb{R}$ is weakly lower semicontinuous. 
Then there exists $z \in C$ such that

$$
\begin{aligned}
& g(z, y)+\langle A z, \eta(y, z)\rangle+\psi(y)-\psi(z) \\
& +\frac{1}{r}\langle\nabla f(z)-\nabla f(x), y-z\rangle \geq 0 \quad \forall y \in C .
\end{aligned}
$$

Proof. Define two set-valued mappings $H, W: C \rightarrow 2^{E}$ as follows:

$$
\begin{array}{r}
H(y)=\{z \in C: g(z, y)+\langle A z, \eta(y, z)\rangle+\psi(y) \\
\left.-\psi(z)+\frac{1}{r}\langle\nabla f(z)-\nabla f(x), y-z\rangle \geq 0\right\} \\
\forall y \in C, \\
W(y)=\{z \in C: g(z, y)+\langle A y, \eta(y, z)\rangle+\psi(y) \\
\left.-\psi(z)+\frac{1}{r}\langle\nabla f(z)-\nabla f(x), y-z\rangle \geq \alpha(y-z)\right\} \\
\forall y \in C .
\end{array}
$$

We claim $H$ is a KKM mapping. By contradiction suppose then there do not exist $\left\{y_{1}, y_{2}, \ldots, y_{n}\right\} \subset C$ and $t_{i}>0(i=$ $1,2, \ldots, n)$ such that $\sum_{i=1}^{n} t_{i}=1$ and $y=\sum_{i=1}^{n} t_{i} y_{i} \notin$ $\bigcup_{i=1}^{n} H\left(y_{i}\right)$. This implies

$$
\begin{gathered}
g\left(y, y_{i}\right)+\left\langle A y, \eta\left(y_{i}, y\right)\right\rangle+\psi\left(y_{i}\right)-\psi(y) \\
+\frac{1}{r}\left\langle\nabla f(y)-\nabla f(x), y-y_{i}\right\rangle<0
\end{gathered}
$$

$$
\forall i=1,2, \ldots, n \text {. }
$$

It follows that

$$
\begin{aligned}
0 & =g(y, y)+\langle A y, \eta(y, y)\rangle=g\left(y, \sum_{i=1}^{n} t_{i} y_{i}\right) \\
& +\left\langle A y, \eta\left(\sum_{i=1}^{n} t_{i} y_{i}, y\right)\right\rangle \leq \sum_{i=1}^{n} t_{i} g\left(y, y_{i}\right) \\
& +\sum_{i=1}^{n} t_{i}\left\langle A y, \eta\left(y_{i}, y\right)\right\rangle<\sum_{i=1}^{n} t_{i}\left(\psi(y)-\psi\left(y_{i}\right)\right. \\
& \left.+\frac{1}{r}\left\langle\nabla f(y)-\nabla f(x), y_{i}-y\right\rangle\right)=\psi(y) \\
& -\sum_{i=1}^{n} t_{i} \psi\left(y_{i}\right) \leq \psi(y)-\psi\left(\sum_{i=1}^{n} t_{i} y_{i}\right)=0,
\end{aligned}
$$

which is a contradiction. Thus $H$ is a KKM mapping.

Next we show that $\forall y \in C H(y) \subset W(y)$.

Let $z \in H(y)$. Then

$$
\begin{gathered}
g(z, y)+\langle A z, \eta(y, z)\rangle+\psi(y)-\psi(z) \\
+\frac{1}{r}\langle\nabla f(z)-\nabla f(x), y-z\rangle \geq 0 .
\end{gathered}
$$

Since $A$ is relaxed $\eta-\alpha$ monotone, we have

$$
\begin{aligned}
& g(z, y)+\langle A y, \eta(y, z)\rangle+\psi(y)-\psi(z) \\
& \quad+\frac{1}{r}\langle\nabla f(z)-\nabla f(x), y-z\rangle \geq g(z, y) \\
& \quad+\langle A z, \eta(y, z)\rangle+\psi(y)-\psi(z) \\
& \quad+\frac{1}{r}\langle\nabla f(z)-\nabla f(x), y-z\rangle+\alpha(y-z) \\
& \quad \geq \alpha(y-z) .
\end{aligned}
$$

Showing that $z \in W(y)$ for all $y \in C$. By Remark 4 it follows that $W$ is a KKM mapping.

We claim also that $W(y)$ is closed in the weak topology of $E$. Let $y \in C$ and $\bar{z}$ be the weak closure point of $W(y)$. Since $E$ is reflexive, there exists a sequence $\left\{z_{n}\right\} \subset W(y)$ such that $z_{n} \rightarrow \bar{z} \in C$ as $n \rightarrow \infty$. Observe that

$$
\begin{aligned}
& g\left(z_{n}, y\right)+\left\langle A y, \eta\left(y, z_{n}\right)\right\rangle+\psi(y)-\psi\left(z_{n}\right) \\
& \quad+\frac{1}{r}\left\langle\nabla f\left(z_{n}\right)-\nabla f(x), y-z_{n}\right\rangle \geq \alpha\left(y-z_{n}\right)
\end{aligned}
$$

is equivalent to

$$
\begin{aligned}
& \frac{1}{r}\left\langle\nabla f\left(z_{n}\right)-\nabla f(x), y-z_{n}\right\rangle \\
& \geq g\left(y, z_{n}\right)+\left\langle A y, \eta\left(z_{n}, y\right)\right\rangle+\psi\left(z_{n}\right)-\psi(y) \\
& \quad+\alpha\left(y-z_{n}\right) .
\end{aligned}
$$

By (iii) and (iv) and taking lim inf as $n \rightarrow \infty$ of both sides of (49) we obtain

$$
\begin{aligned}
& \frac{1}{r}\langle\nabla f(\bar{z})-\nabla f(x), y-\bar{z}\rangle \\
& \geq g(y, \bar{z})+\langle A y, \eta(\bar{z}, y)\rangle+\psi(\bar{z})-\psi(y) \\
& \quad+\alpha(y-\bar{z}) .
\end{aligned}
$$

That is, $\bar{z} \in W(y) \forall y \in C$. This implies $W(y)$ is weakly closed for all $y \in C$. Since $C$ is weakly compact, then $W(y)$ is weakly compact in $C$ for all $y \in C$.

It is clear that the solution sets of problem (34) and (35) are $\bigcap_{y \in C} H(y)$ and $\bigcap_{y \in C} W(y)$. Using Lemmas 16 and 5 we obtain

$$
\bigcap_{y \in C} H(y)=\bigcap_{y \in C} W(y) \neq \emptyset
$$

Hence there exists $z \in C$ such that

$$
\begin{aligned}
& g(z, y)+\langle A z, \eta(y, z)\rangle+\psi(y)-\psi(z) \\
&+\frac{1}{r}\langle\nabla f(z)-\nabla f(x), y-z\rangle \geq 0 \quad \forall y \in C .
\end{aligned}
$$

This completes the proof. 
Lemma 18. Let $E$ be a reflexive Banach space with the dual $E^{*}$ and let $C$ be a nonempty closed, convex, and bounded subset of $E$. Let $f: E \rightarrow(-\infty,+\infty]$ be Legendre and Gâteaux differentiable function. Let $A: C \rightarrow E^{*}$ be $\eta$-hemicontinuous and relaxed $\eta-\alpha$ monotone mapping and $g: C \times C \rightarrow \mathbb{R}$ be a bifunction satisfying (C1), (C2), and (C4). Let $\psi: C \rightarrow \mathbb{R}$ be proper, convex, and lower semicontinuous. For $r>0$ and $x \in E$, define a map $T_{r}: E \rightarrow 2^{C}$ by

$$
\begin{aligned}
& T_{r}(x)=\{z \in C: g(z, y)+\langle A y, \eta(y z)\rangle+\psi(y) \\
& \left.\quad-\psi(z)+\frac{1}{r}\langle\nabla f(z)-\nabla f(x), y-z\rangle \geq 0 \forall y \in C\right\} .
\end{aligned}
$$

\section{Assume that}

(i) $\eta(z, y)+\eta(y, z)=0 \forall z, y \in C$;

(ii) $\langle A u, \eta(\cdot, v)\rangle$ is convex and lower semicontinuous for fixed $u, v \in C$;

(iii) $\alpha: E \rightarrow \mathbb{R}$ is weakly lower semicontinuous;

(iv) $\alpha(x-y)+\alpha(y-z) \geq 0 \forall x, y \in C$.

Then

(1) $T_{r}$ is single-valued;

(2) $T_{r}$ is a Bregman firmly nonexpansive type mapping; that is,

$$
\begin{aligned}
& \left\langle\nabla f\left(T_{r} x\right)-\nabla f\left(T_{r} y\right), T_{r} x-T_{r} y\right\rangle \\
& \quad \leq\left\langle\nabla f(x)-\nabla f(y), T_{r} x-T_{r} y\right\rangle \quad \forall x, y \in C ;
\end{aligned}
$$

(3) $F\left(T_{r}\right)=E P(g, A)$;

(4) $T_{r}$ is a Bregman quasi nonexpansive satisfying

$$
D_{f}\left(u, T_{r} x\right)+D_{f}\left(T_{r} x, x\right) \leq D_{f}(u, x)
$$

(5) $E P(g, A)$ is closed and convex.

Proof. First we show that $T_{r}$ is single-valued. Let $z_{1}, z_{2} \in T_{r} x$; then

$$
\begin{aligned}
& g\left(z_{1}, z_{2}\right)+\left\langle A z_{1}, \eta\left(z_{2}, z_{1}\right)\right\rangle+\psi\left(z_{2}\right)-\psi\left(z_{1}\right) \\
& +\frac{1}{r}\left\langle\nabla f\left(z_{1}\right)-\nabla f(x), z_{2}-z_{1}\right\rangle \geq 0, \\
& g\left(z_{2}, z_{1}\right)+\left\langle A z_{2}, \eta\left(z_{1}, z_{2}\right)\right\rangle+\psi\left(z_{1}\right)-\psi\left(z_{2}\right) \\
& +\frac{1}{r}\left\langle\nabla f\left(z_{2}\right)-\nabla f(x), z_{1}-z_{2}\right\rangle \geq 0 .
\end{aligned}
$$

By using (C2), adding (56) yields

$$
\begin{aligned}
& \left\langle A z_{1}, \eta\left(z_{2}, z_{1}\right)\right\rangle+\left\langle A z_{2}, \eta\left(z_{1}, z_{2}\right)\right\rangle \\
& \quad+\frac{1}{r}\left\langle\nabla f\left(z_{1}\right)-\nabla f\left(z_{2}\right), z_{2}-z_{1}\right\rangle \geq 0 .
\end{aligned}
$$

By (i) we have

$$
\begin{aligned}
\left\langle A z_{1}\right. & \left.-A z_{2}, \eta\left(z_{2}, z_{1}\right)\right\rangle \\
& +\frac{1}{r}\left\langle\nabla f\left(z_{1}\right)-\nabla f\left(z_{2}\right), z_{2}-z_{1}\right\rangle \geq 0 .
\end{aligned}
$$

Since $A$ is relaxed $\eta-\alpha$ monotone, we obtain

$$
\begin{aligned}
& \frac{1}{r}\left\langle\nabla f\left(z_{1}\right)-\nabla f\left(z_{2}\right), z_{2}-z_{1}\right\rangle \\
& \quad \geq\left\langle A z_{2}-A z_{1}, \eta\left(z_{2}, z_{1}\right)\right\rangle \geq \alpha\left(z_{2}-z_{1}\right) .
\end{aligned}
$$

Thus

$$
\left\langle\nabla f\left(z_{1}\right)-\nabla f\left(z_{2}\right), z_{2}-z_{1}\right\rangle \geq r \alpha\left(z_{2}-z_{1}\right) .
$$

Interchanging $z_{1}$ and $z_{2}$ in (60), we have

$$
\left\langle\nabla f\left(z_{2}\right)-\nabla f\left(z_{1}\right), z_{1}-z_{2}\right\rangle \geq r \alpha\left(z_{1}-z_{2}\right) .
$$

Adding (60) and (61), we have

$$
\begin{aligned}
&\left\langle\nabla f\left(z_{1}\right)-\nabla f\left(z_{2}\right), z_{2}-z_{1}\right\rangle \\
& \quad+\left\langle\nabla f\left(z_{2}\right)-\nabla f\left(z_{1}\right), z_{1}-z_{2}\right\rangle \\
& \geq \\
& \quad r\left(\alpha\left(z_{2}-z_{1}\right)+\alpha\left(z_{1}-z_{2}\right)\right) .
\end{aligned}
$$

Hence

$$
\begin{aligned}
2\left\langle\nabla f\left(z_{1}\right)-\nabla f\left(z_{2}\right), z_{2}-z_{1}\right\rangle \\
\quad \geq r\left(\alpha\left(z_{2}-z_{1}\right)+\alpha\left(z_{1}-z_{2}\right)\right) .
\end{aligned}
$$

By (iv), we have

$$
\left\langle\nabla f\left(z_{1}\right)-\nabla f\left(z_{2}\right), z_{2}-z_{1}\right\rangle \geq 0 .
$$

Thus,

$$
\left\langle\nabla f\left(z_{2}\right)-\nabla f\left(z_{1}\right), z_{2}-z_{1}\right\rangle \leq 0 .
$$

Since $f$ is convex and Gâteaux differentiable we have

$$
\left\langle\nabla f\left(z_{2}\right)-\nabla f\left(z_{1}\right), z_{2}-z_{1}\right\rangle \geq 0 .
$$

By (65) and (66) we obtain

$$
\left\langle\nabla f\left(z_{2}\right)-\nabla f\left(z_{1}\right), z_{2}-z_{1}\right\rangle=0 .
$$

Since $f$ is Legendre function, then $z_{1}=z_{2}$.

Next we show that $T_{r}$ is Bregman firmly nonexpansive type. Let $x, y \in C$; then

$$
\begin{aligned}
g & \left(T_{r} x, T_{r} y\right)+\left\langle A T_{r} x, \eta\left(T_{r} y, T_{r} x\right)\right\rangle+\psi\left(T_{r} y\right) \\
& -\psi\left(T_{r} x\right) \\
& +\frac{1}{r}\left\langle\nabla f\left(T_{r} x\right)-\nabla f(x), T_{r} y-T_{r} x\right\rangle \geq 0, \\
g & \left(T_{r} y, T_{r} x\right)+\left\langle A T_{r} y, \eta\left(T_{r} x, T_{r} y\right)\right\rangle+\psi\left(T_{r} x\right) \\
& -\psi\left(T_{r} y\right) \\
& +\frac{1}{r}\left\langle\nabla f\left(T_{r} y\right)-\nabla f(y), T_{r} x-T_{r} y\right\rangle \geq 0 .
\end{aligned}
$$


Adding (68), using (i) and (C2), we obtain

$$
\begin{aligned}
& \left\langle A T_{r} x-A T_{r} y, \eta\left(T_{r} y, T_{r} x\right)\right\rangle+\frac{1}{r}\left\langle\nabla f\left(T_{r} x\right)\right. \\
& \left.-\nabla f\left(T_{r} y\right)-\nabla f(x)+\nabla f(y), T_{r} y-T_{r} x\right\rangle \geq 0,
\end{aligned}
$$

so that

$$
\begin{gathered}
\frac{1}{r}\left\langle\nabla f\left(T_{r} x\right)-\nabla f\left(T_{r} y\right)-\nabla f(x)+\nabla f(y), T_{r} y\right. \\
\left.-T_{r} x\right\rangle \geq\left\langle A T_{r} y-A T_{r} x, \eta\left(T_{r} y, T_{r} x\right)\right\rangle .
\end{gathered}
$$

Since $A$ is relaxed $\eta-\alpha$ monotone and $r>0$, we have

$$
\begin{aligned}
& \left\langle\nabla f\left(T_{r} x\right)-\nabla f\left(T_{r} y\right)-\nabla f(x)+\nabla f(y), T_{r} y-T_{r} x\right\rangle \\
& \quad \geq r \alpha\left(T_{r} y-T_{r} x\right) .
\end{aligned}
$$

Also interchanging the roles of $x$ and $y$ in (71) and applying (iv), we have

$$
\begin{aligned}
& 2\left\langle\nabla f\left(T_{r} x\right)-\nabla f\left(T_{r} y\right)-\nabla f(x)+\nabla f(y), T_{r} y\right. \\
& \left.-T_{r} x\right\rangle \geq 0 .
\end{aligned}
$$

Hence,

$$
\begin{aligned}
& \left\langle\nabla f\left(T_{r} x\right)-\nabla f\left(T_{r} y\right), T_{r} x-T_{r} y\right\rangle \\
& \quad \leq\left\langle\nabla f(x)-\nabla f(y), T_{r} x-T_{r} y\right\rangle,
\end{aligned}
$$

showing that $T_{r}$ is Bregman firmly nonexpansive type.

We now show that $F\left(T_{r}\right)=\operatorname{EP}(g, A)$. Indeed

$$
\begin{aligned}
& u \in F\left(T_{r}\right) \Longleftrightarrow u \in T_{r} u \Longleftrightarrow \\
& g(u, y)+\langle A u, \eta(y, u)\rangle+\psi(y)-\psi(u) \\
&+\frac{1}{r}\langle\nabla f(u)-\nabla f(u), y-u\rangle \geq 0 \quad \forall y \in C \Longleftrightarrow \\
& g(u, y)+\langle A u, \eta(y, u)\rangle+\psi(y)-\psi(u) \geq 0 \\
& \forall y \in C \Longleftrightarrow
\end{aligned}
$$

$$
u \in \operatorname{EP}(g, A)
$$

Next we prove that $T_{r}$ is Bregman quasi nonexpansive mapping.

Since

$$
\begin{aligned}
D_{f} & \left(T_{r} x, T_{r} y\right)+D_{f}\left(T_{r} y, T_{r} x\right) \\
\leq & D_{f}\left(T_{r} x, y\right)+D_{f}\left(T_{r} y, x\right)-D_{f}\left(T_{r} x, x\right) \\
& \quad-D_{f}\left(T_{r} y, y\right),
\end{aligned}
$$

we have

$$
\begin{aligned}
& D_{f}\left(T_{r} x, T_{r} y\right)+D_{f}\left(T_{r} y, T_{r} x\right) \\
& \leq D_{f}\left(T_{r} x, y\right)+D_{f}\left(T_{r} y, x\right) .
\end{aligned}
$$

Let $u=y \in F\left(T_{r}\right)$; then from (76) we obtain

$$
D_{f}\left(u, T_{r} x\right) \leq D_{f}(u, x) .
$$

This shows that $T_{r}$ is Bregman quasi nonexpansive mapping which is Bregman quasiasymptotically nonexpansive. Also from (75), we have

$$
\begin{aligned}
& D_{f}\left(T_{r} x, T_{r} y\right)+D_{f}\left(T_{r} y, T_{r} x\right)+D_{f}\left(T_{r} x, x\right) \\
& \quad+D_{f}\left(T_{r} y, y\right) \leq D_{f}\left(T_{r} x, y\right)+D_{f}\left(T_{r} y, x\right) .
\end{aligned}
$$

As $u=y \in F\left(T_{r}\right)$, we obtain

$$
D_{f}\left(u, T_{r} x\right)+D_{f}\left(T_{r} x, x\right) \leq D_{f}(u, x)
$$

Lastly, using (3), (4), and Lemma 14 we obtain that $\operatorname{EP}(g, A)$ is closed and convex.

Definition 19. Let $C$ be a nonempty, closed, and convex subset of a real Banach space $E$. Let $\left\{T_{i}\right\}_{i=1}^{N}$ be a finite family of Bregman quasiasymptotically nonexpansive mappings. For any $n \in \mathbb{N}$, define a mapping $K_{n}: C \rightarrow C$ as follows:

$$
\begin{aligned}
& S_{n, 0} x=x \\
& S_{n, 1} x=P_{C}^{f}\left(\nabla f^{*}\left(\alpha_{n, 1} \nabla f\left(T_{1}^{n} x\right)+\left(1-\alpha_{n, 1}\right) \nabla f(x)\right)\right) \\
& S_{n, 2} x=P_{C}^{f}\left(\nabla f ^ { * } \left(\alpha_{n, 2} \nabla f\left(T_{2}^{n} S_{n, 1} x\right)\right.\right. \\
& \left.\left.+\left(1-\alpha_{n, 2}\right) \nabla f\left(S_{n, 1} x\right)\right)\right) \\
& S_{n, 3} x=P_{C}^{f}\left(\nabla f ^ { * } \left(\alpha_{n, 3} \nabla f\left(T_{3}^{n} S_{n, 2} x\right)\right.\right. \\
& \left.\left.+\left(1-\alpha_{n, 3}\right) \nabla f\left(S_{n, 2} x\right)\right)\right) \\
& S_{n, N-1} x=P_{C}^{f}\left(\nabla f ^ { * } \left(\alpha_{n, N-1} \nabla f\left(T_{N-1}^{n} S_{n, N-2} x\right)\right.\right. \\
& \left.\left.+\left(1-\alpha_{n, N-1}\right) \nabla f\left(S_{n, N-2} x\right)\right)\right) \\
& K_{n} x=S_{n, N} x=P_{C}^{f}\left(\nabla f ^ { * } \left(\alpha_{n, N} \nabla f\left(T_{N}^{n} S_{n, N-1} x\right)\right.\right. \\
& \left.\left.+\left(1-\alpha_{n, N}\right) \nabla f\left(S_{n, N-1} x\right)\right)\right) .
\end{aligned}
$$

Such a mapping $K_{n}$ is called the Bregman $K$-mapping generated by $T_{1}, T_{2}, T_{3}, \ldots, T_{N}$ and $\alpha_{n, i} \in(0,1), i=1,2,3, \ldots, N$.

Using the above definition, we have the following Lemma.

Lemma 20. Let $E$ be a reflexive Banach space with the dual $E^{*}$ and let $C$ be a nonempty, closed, convex, and bounded subset of $E$. Let $f: E \rightarrow(-\infty,+\infty]$ be strongly coercive, Legendre, uniformly Fréchet differentiable, and totally convex function which is bounded on bounded subsets of E. Let $\left\{T_{i}\right\}_{i=1}^{N}$ be a finite 
family of continuous Bregman quasiasymptotically nonexpansive mappings of $C$ into itself such that $\bigcap_{i=1}^{N} F\left(T_{i}\right) \neq \emptyset$. Let $\left\{\alpha_{n, i}\right\}$ be a real sequence in $(0,1)$ such that $\lim _{i n f} \operatorname{in}_{n \rightarrow \infty} \alpha_{n, i}>0 \forall i \in$ $\{1,2,3, \ldots, N\}$. Let $K_{n}$ be Bregman $K$-mapping generated by $T_{1}, T_{2}, T_{3}, \ldots, T_{N}$ in (80). Then

(i) $D_{f}\left(x^{*}, K_{n} x\right) \leq\left(1+t_{n}\right) D_{f}\left(x^{*}, x\right)$ for all $x^{*} \in F\left(K_{n}\right)$ and $x \in C$, where $t_{n} \rightarrow 0$ as $n \rightarrow \infty$;

(ii) $F\left(K_{n}\right)=\bigcap_{i=1}^{N} F\left(T_{i}\right)$;

(iii) $K_{n}$ is a closed mapping.

Proof. Let $x^{*} \in F\left(K_{n}\right)$ and $x \in C$. Then by Lemma 13(ii) and inequality (16) we have

$$
\begin{aligned}
& D_{f}\left(x^{*}, K_{n} x\right) \\
& =D_{f}\left(x^{*}, P_{C}^{f}\left(\nabla f ^ { * } \left(\alpha_{n, N} \nabla f\left(T_{N}^{n} S_{n, N-1} x\right)\right.\right.\right. \\
& \left.\left.\left.+\left(1-\alpha_{n, N}\right) \nabla f\left(S_{n, N-1} x\right)\right)\right)\right) \\
& \leq D_{f}\left(x^{*}, \nabla f^{*}\left(\alpha_{n, N} \nabla f\left(T_{N}^{n} S_{n, N-1} x\right)+\left(1-\alpha_{n, N}\right) \nabla f\right.\right. \\
& \left.\left.\cdot\left(S_{n, N-1} x\right)\right)\right) \\
& \leq \alpha_{n, N} D_{f}\left(x^{*}, T_{N}^{n} S_{n, N-1} x\right)+\left(1-\alpha_{n, N}\right) D_{f}\left(x^{*},\right. \\
& \left.S_{n, N-1} x\right) \\
& \leq \alpha_{n, N}\left(1+v_{n, N}\right) D_{f}\left(x^{*}, S_{n, N-1} x\right)+\left(1-\alpha_{n, N}\right) \\
& \text { - } D_{f}\left(x^{*}, S_{n, N-1} x\right) \\
& =\left(1+\alpha_{n, N} v_{n, N}\right) D_{f}\left(x^{*}, S_{n, N-1} x\right) \\
& =\left(1+\alpha_{n, N} v_{n, N}\right) D_{f}\left(x^{*}, \nabla f^{*}\left(\alpha_{n, N-1} \nabla f\right.\right. \\
& \left.\left.\cdot\left(T_{N-1}^{n} S_{n, N-2} x\right)+\left(1-\alpha_{n, N-1}\right) \nabla f\left(S_{n, N-2} x\right)\right)\right) \\
& \leq\left(1+\alpha_{n, N} v_{n, N}\right)\left[\alpha_{n, N-1} D_{f}\left(x^{*}, T_{N-1}^{n} S_{n, N-2} x\right)+(1\right. \\
& \left.\left.-\alpha_{n, N-1}\right) D_{f}\left(x^{*}, S_{n, N-2} x\right)\right] \\
& \leq\left(1+\alpha_{n, N} v_{n, N}\right)\left[\alpha_{n, N-1}\left(1+v_{n, N-1}\right) D_{f}\left(x^{*}, S_{n, N-2} x\right)\right. \\
& \left.+\left(1-\alpha_{n, N-1}\right) D_{f}\left(x^{*}, S_{n, N-2} x\right)\right] \\
& =\left(1+\alpha_{n, N} v_{n, N}\right)\left(1+\alpha_{n, N-1} v_{n, N-1}\right) D_{f}\left(x^{*}, S_{n, N-2} x\right) \\
& \leq\left(1+\alpha_{n, N} v_{n, N}\right)\left(1+\alpha_{n, N-1} v_{n, N-1}\right)\left(1+\alpha_{n, N-2} v_{n, N-2}\right) \\
& \text { - } D_{f}\left(x^{*}, S_{n, N-3} x\right) \\
& \leq\left(1+\alpha_{n, N} v_{n, N}\right)\left(1+\alpha_{n, N-1} v_{n, N-1}\right) \cdots\left(1+\alpha_{n, 2} v_{n, 2}\right) \\
& \cdot D_{f}\left(x^{*}, S_{n, 1} x\right) \text {. }
\end{aligned}
$$

Hence,

$$
\begin{aligned}
D_{f} & \left(x^{*}, K_{n} x\right) \leq\left(1+\alpha_{n, N} v_{n, N}\right) \\
\cdot & \left(1+\alpha_{n, N-1} v_{n, N-1}\right) \cdots\left(1+\alpha_{n, 2} v_{n, 2}\right) \\
\cdot & D_{f}\left(x^{*}, \nabla f^{*}\left(\alpha_{n, 1} \nabla f\left(T_{1}^{n} x\right)+\left(1-\alpha_{n, 1}\right) \nabla f(x)\right)\right) \\
& \leq\left(1+\alpha_{n, N} v_{n, N}\right) \\
& \cdot\left(1+\alpha_{n, N-1} v_{n, N-1}\right) \cdots\left(1+\alpha_{n, 1} v_{n, 1}\right) D_{f}\left(x^{*}, x\right) \\
& =\prod_{i=1}^{N}\left(1+\alpha_{n, i} v_{n, i}\right) D_{f}\left(x^{*}, x\right) .
\end{aligned}
$$

Observe that $\alpha_{n, i} v_{n, i} \rightarrow 0$ as $n \rightarrow \infty$ for all $i \in\{1,2,3, \ldots, N\}$. Let $\prod_{i=1}^{N}\left(1+\alpha_{n, i} v_{n, i}\right)=\left(1+t_{n}\right)$; then $t_{n} \rightarrow 0$ as $n \rightarrow \infty$ and

$$
D_{f}\left(x^{*}, K_{n} x\right) \leq\left(1+t_{n}\right) D_{f}\left(x^{*}, x\right) \text {. }
$$

Next we show that $F\left(K_{n}\right)=\bigcap_{i=1}^{N} F\left(T_{i}\right)$. It is obvious that $\bigcap_{i=1}^{N} F\left(T_{i}\right) \subset F\left(K_{n}\right)$. Now let $z \in F\left(K_{n}\right)$ and $x^{*}$ be any point in $\bigcap_{i=1}^{N} F\left(T_{i}\right)$.

Let $r_{1}=\sup \left\{\|\nabla f(z)\|,\left\|\nabla f\left(S_{n, i} z\right)\right\|,\left\|\nabla f\left(T_{i}^{n} S_{n, i} z\right)\right\|\right\}$ for $1 \leq$ $i \leq N$. Then

$$
\begin{aligned}
& D_{f}\left(x^{*}, z\right)=D_{f}\left(x^{*}, K_{n} z\right)=D_{f}\left(x^{*},\right. \\
& \quad P_{C}^{f}\left(\nabla f ^ { * } \left(\alpha_{n, N} \nabla f\left(T_{N}^{n} S_{n, N-1} z\right)\right.\right. \\
& \left.\left.\left.+\left(1-\alpha_{n, N}\right) \nabla f\left(S_{n, N-1} z\right)\right)\right)\right) \leq D_{f}\left(x^{*},\right. \\
& \nabla f^{*}\left(\alpha_{n, N} \nabla f\left(T_{N}^{n} S_{n, N-1} z\right)+\left(1-\alpha_{n, N}\right) \nabla f\right. \\
& \left.\left.\cdot\left(S_{n, N-1} z\right)\right)\right)=V_{f}\left(x^{*}, \alpha_{n, N} \nabla f\left(T_{N}^{n} S_{n, N-1} z\right)\right. \\
& \left.+\left(1-\alpha_{n, N}\right) \nabla f\left(S_{n, N-1} z\right)\right)=f\left(x^{*}\right)-\left\langle x^{*}, \alpha_{n, N} \nabla f\right. \\
& \left.\cdot\left(T_{N}^{n} S_{n, N-1} z\right)+\left(1-\alpha_{n, N}\right) \nabla f\left(S_{n, N-1} z\right)\right\rangle \\
& +f^{*}\left(\alpha_{n, N} \nabla f\left(T_{N}^{n} S_{n, N-1} z\right)+\left(1-\alpha_{n, N}\right) \nabla f\right. \\
& \left.\cdot\left(S_{n, N-1} z\right)\right) .
\end{aligned}
$$

Since $f$ is uniformly Fréchet differentiable function which is bounded on bounded subsets of $E$, then by Lemma $10 \nabla f$ is uniformly continuous on bounded subsets and consequently from Lemma $11 f^{*}$ is uniformly convex. Therefore in view of Lemma 9 we have

$$
\begin{aligned}
D_{f} & \left(x^{*}, z\right) \leq f\left(x^{*}\right)-\left\langle x^{*}, \alpha_{n, N} \nabla f\left(T_{N}^{n} S_{n, N-1} z\right)\right. \\
+ & \left.\left(1-\alpha_{n, N}\right) \nabla f\left(S_{n, N-1} z\right)\right\rangle \\
+ & \alpha_{n, N} f^{*}\left(\nabla f\left(T_{N}^{n} S_{n, N-1} z\right)\right)+\left(1-\alpha_{n, N}\right) \\
& \cdot f^{*}\left(\nabla f\left(S_{n, N-1} z\right)\right)-\alpha_{n, N}\left(1-\alpha_{n, N}\right) \\
& \cdot \rho_{r_{1}}^{*}\left(\left\|\nabla f\left(T_{N}^{n} S_{n, N-1} z\right)-\nabla f\left(S_{n, N-1} z\right)\right\|\right)
\end{aligned}
$$




$$
\begin{aligned}
& =\alpha_{n, N}\left(f\left(x^{*}\right)-\left\langle x^{*}, \nabla f\left(T_{N}^{n} S_{n, N-1} z\right)\right\rangle\right. \\
& \left.+f^{*}\left(\nabla f\left(T_{N}^{n} S_{n, N-1} z\right)\right)\right)+\left(1-\alpha_{n, N}\right)\left(f\left(x^{*}\right)\right. \\
& \left.-\left\langle x^{*}, \nabla f\left(S_{n, N-1} z\right)\right\rangle+f^{*}\left(\nabla f\left(S_{n, N-1} z\right)\right)\right) \\
& -\alpha_{n, N}\left(1-\alpha_{n, N}\right) \\
& \cdot \rho_{r_{1}}^{*}\left(\left\|\nabla f\left(T_{N}^{n} S_{n, N-1} z\right)-\nabla f\left(S_{n, N-1} z\right)\right\|\right) \\
& =\alpha_{n, N} V_{f}\left(x^{*}, \nabla f\left(T_{N}^{n} S_{n, N-1} z\right)\right)+\left(1-\alpha_{n, N}\right) \\
& \cdot V_{f}\left(x^{*}, \nabla f\left(S_{n, N-1} z\right)\right)-\alpha_{n, N}\left(1-\alpha_{n, N}\right) \\
& \cdot \rho_{r_{1}}^{*}\left(\left\|\nabla f\left(T_{N}^{n} S_{n, N-1} z\right)-\nabla f\left(S_{n, N-1} z\right)\right\|\right) \\
& =\alpha_{n, N} D_{f}\left(x^{*}, T_{N}^{n} S_{n, N-1} z\right)+\left(1-\alpha_{n, N}\right) \\
& \cdot D_{f}\left(x^{*}, S_{n, N-1} z\right)-\alpha_{n, N}\left(1-\alpha_{n, N}\right) \\
& \text { - } \rho_{r_{1}}^{*}\left(\left\|\nabla f\left(T_{N}^{n} S_{n, N-1} z\right)-\nabla f\left(S_{n, N-1} z\right)\right\|\right) \leq \alpha_{n, N}(1 \\
& \left.+v_{n, N}\right) D_{f}\left(x^{*}, S_{n, N-1} z\right)+\left(1-\alpha_{n, N}\right) \\
& \cdot D_{f}\left(x^{*}, S_{n, N-1} z\right)-\alpha_{n, N}\left(1-\alpha_{n, N}\right) \\
& \cdot \rho_{r_{1}}^{*}\left(\left\|\nabla f\left(T_{N}^{n} S_{n, N-1} z\right)-\nabla f\left(S_{n, N-1} z\right)\right\|\right)=(1 \\
& \left.+\alpha_{n, N} v_{n, N}\right) D_{f}\left(x^{*}, S_{n, N-1} z\right)-\alpha_{n, N}\left(1-\alpha_{n, N}\right) \\
& \cdot \rho_{r_{1}}^{*}\left(\left\|\nabla f\left(T_{N}^{n} S_{n, N-1} z\right)-\nabla f\left(S_{n, N-1} z\right)\right\|\right) \text {. }
\end{aligned}
$$

Continuing in this fashion we obtain

$$
\begin{aligned}
& D_{f}\left(x^{*}, z\right) \leq\left(1+\alpha_{n, N} v_{n, N}\right) \\
& \cdot\left(1+\alpha_{n, N-1} v_{n, N-1}\right) \cdots\left(1+\alpha_{n, 1} v_{n, 1}\right) D_{f}\left(x^{*}, z\right) \\
& -\left(1+\alpha_{n, N} v_{n, N}\right) \\
& \cdot\left(1+\alpha_{n, N-1} v_{n, N-1}\right) \cdots\left(1+\alpha_{n, 2} v_{n, 2}\right) \alpha_{n, 1}\left(1-\alpha_{n, 1}\right) \\
& \cdot \rho_{r_{1}}^{*}\left(\left\|\nabla f\left(T_{1}^{n} z\right)-\nabla f(z)\right\|\right)-\left(1+\alpha_{n, N} v_{n, N}\right) \\
& \cdot\left(1+\alpha_{n, N-1} v_{n, N-1}\right) \cdots\left(1+\alpha_{n, 3} v_{n, 3}\right) \alpha_{n, 2}\left(1-\alpha_{n, 2}\right) \\
& \cdot \rho_{r_{1}}^{*}\left(\left\|\nabla f\left(T_{2}^{n} S_{n, 1} z\right)-\nabla f\left(S_{n, 1} z\right)\right\|\right) \\
& -\left(1+\alpha_{n, N} v_{n, N}\right) \\
& \cdot\left(1+\alpha_{n, N-1} v_{n, N-1}\right) \cdots\left(1+\alpha_{n, 4} v_{n, 4}\right) \alpha_{n, 3}\left(1-\alpha_{n, 3}\right) \\
& \cdot \rho_{r_{1}}^{*}\left(\left\|\nabla f\left(T_{3}^{n} S_{n, 2} z\right)-\nabla f\left(S_{n, 2} z\right)\right\|\right) \\
& -\left(1+\alpha_{n, N} v_{n, N}\right) \alpha_{n, N-1}\left(1-\alpha_{n, N-1}\right) \\
& \vdots \\
&
\end{aligned}
$$

$$
\begin{aligned}
& \cdot \rho_{r_{1}}^{*}\left(\left\|\nabla f\left(T_{N-1}^{n} S_{n, N-2} z\right)-\nabla f\left(S_{n, N-2} z\right)\right\|\right) \\
& -\alpha_{n, N}\left(1-\alpha_{n, N}\right) \\
& \cdot \rho_{r_{1}}^{*}\left(\left\|\nabla f\left(T_{N}^{n} S_{n, N-1} z\right)-\nabla f\left(S_{n, N-1} z\right)\right\|\right) .
\end{aligned}
$$

From (86), we have

$$
\begin{aligned}
(1+ & \left.\alpha_{n, N} v_{n, N}\right)\left(1+\alpha_{n, N-1} v_{n, N-1}\right) \cdots\left(1+\alpha_{n, 2} v_{n, 2}\right) \\
& \cdot \alpha_{n, 1}\left(1-\alpha_{n, 1}\right) \rho_{r_{1}}^{*}\left(\left\|\nabla f\left(T_{1}^{n} z\right)-\nabla f(z)\right\|\right) \\
& \leq\left(1+\alpha_{n, N} v_{n, N}\right) \\
& \cdot\left(1+\alpha_{n, N-1} v_{n, N-1}\right) \cdots\left(1+\alpha_{n, 1} v_{n, 1}\right) D_{f}\left(x^{*}, z\right) \\
& -D_{f}\left(x^{*}, z\right) .
\end{aligned}
$$

Since liminf $\alpha_{n, 1}\left(1-\alpha_{n, 1}\right)>0$, we have

$$
\lim _{n \rightarrow \infty} \rho_{r_{1}}^{*}\left(\left\|\nabla f\left(T_{1}^{n} z\right)-\nabla f(z)\right\|\right)=0
$$

By the property of $\rho_{r_{1}}^{*}$, we obtain

$$
\lim _{n \rightarrow \infty}\left(\left\|\nabla f\left(T_{1}^{n} z\right)-\nabla f(z)\right\|\right)=0 .
$$

In similar fashion and assuming $\lim \inf \alpha_{n, i}\left(1-\alpha_{n, i}\right)>0 \forall i \epsilon$ $\{2,3, \ldots, N\}$ we have

$$
\begin{gathered}
\lim _{n \rightarrow \infty}\left\|\nabla f\left(T_{2}^{n} S_{n, 1} z\right)-\nabla f\left(S_{n, 1} z\right)\right\| \\
=\lim _{n \rightarrow \infty}\left\|\nabla f\left(T_{3}^{n} S_{n, 2} z\right)-\nabla f\left(S_{n, 2} z\right)\right\| \\
=\lim _{n \rightarrow \infty}\left\|\nabla f\left(T_{4}^{n} S_{n, 3} z\right)-\nabla f\left(S_{n, 3} z\right)\right\| \\
\vdots \\
=\lim _{n \rightarrow \infty}\left\|\nabla f\left(T_{N}^{n} S_{n, N-1} z\right)-\nabla f\left(S_{n, N-1} z\right)\right\|=0 .
\end{gathered}
$$

Since by Lemma $12 \nabla f^{*}$ is uniformly continuous, we obtain from (89) that $T_{1}^{n} z \rightarrow z$ as $n \rightarrow \infty$. By our assumption, $T_{1}$ is continuous and so $T_{1} z=z$, that is, $z \in F\left(T_{1}\right)$. Also from (90), we obtain

$$
\begin{gathered}
T_{2}^{n} S_{n, 1} z=S_{n, 1} z \\
T_{3}^{n} S_{n, 2} z=S_{n, 2} z \\
\vdots \\
T_{N}^{n} S_{n, N-1} z=S_{n, N-1} z .
\end{gathered}
$$

On the other hand

$$
\begin{aligned}
& D_{f}\left(z, S_{n, 1} z\right) \\
& \quad \leq D_{f}\left(z, \nabla f^{*}\left(\alpha_{n, 1} \nabla f\left(T_{1}^{n} x\right)+\left(1-\alpha_{n, 1}\right) \nabla f(x)\right)\right) \\
& \quad \leq \alpha_{n, 1} D_{f}\left(z, T_{1}^{n} z\right)+\left(1-\alpha_{n, 1}\right) D_{f}(z, z) .
\end{aligned}
$$


Since $T_{1}^{n} z=z$ we get

$$
D_{f}\left(z, S_{n, 1} z\right)=0 \quad \text { and so } S_{n, 1} z=z \text {. }
$$

From (91) and (93), we have $z \in F\left(S_{n, 1}\right)$ and $z \in F\left(T_{2}\right)$. Applying the same argument we can conclude that $z \in F\left(T_{i}\right)$ for $i=3,4, \ldots, N$. Thus, it follows that $z \in \bigcap_{i=1}^{N} F\left(T_{i}\right)$.

Next we show that $K_{n}$ is closed.

From (80)

$$
S_{n, 1} x=P_{C}^{f}\left(\nabla f^{*}\left(\alpha_{n, 1} \nabla f\left(T_{1}^{n} x\right)+\left(1-\alpha_{n, 1}\right) \nabla f(x)\right)\right) .
$$

Let $\left\{x_{m}\right\}$ be a sequence in $C$ such that $x_{m} \rightarrow \bar{x}$ and $S_{n, 1} x_{m} \rightarrow$ $y$ as $m \rightarrow \infty$. Since $T_{i}$ is continuous for each $i=1,2,3, \ldots, N$, $\nabla f, \nabla f^{*}$ are uniformly continuous and applying Lemma 15 we have

$$
S_{n, 1} x_{m} \longrightarrow S_{n, 1} \bar{x} \quad \text { as } m \longrightarrow \infty
$$

By uniqueness of limit, we get $S_{n, 1} \bar{x}=y$ showing that $S_{n, 1}$ is closed. Using (95), we have that $S_{n, 2}$ is closed. Continuing in this way we obtain that $K_{n}$ is closed.

Now we prove the strong convergence theorems.

Theorem 21. Let $E$ be a reflexive Banach space with the dual $E^{*}$ and let $C$ be a nonempty, closed, convex, and bounded subset of $E$. Let $f: E \rightarrow(-\infty,+\infty]$ be a strongly coercive, Legendre, uniformly Fréchet differentiable, and totally convex function which is bounded on bounded subsets of $E$. For each $j=1,2,3, \ldots, m$, let $A_{j}: C \rightarrow E^{*}$ be $\eta^{-}$ hemicontinuous and relaxed $\eta-\alpha$ monotone mappings and $g_{j}: C \times C \rightarrow \mathbb{R}$ be bifunctions satisfying $(C 1)-(C 4)$. Let $\psi_{j}: C \rightarrow \mathbb{R}$ be proper, convex, and lower semicontinuous mappings. Let $\left\{T_{i}\right\}_{i=1}^{N}$ be a finite family of continuous Bregman quasiasymptotically nonexpansive mappings of $C$ into itself such that $\mathscr{F}=\left[\bigcap_{i=1}^{N} F\left(T_{i}\right)\right] \cap\left[E P\left(g_{j}, A_{j}\right)\right] \neq \emptyset$. Let $K_{n}$ be the Bregman $K$-mapping generated by $T_{1}, T_{2}, T_{3}, \ldots, T_{N}$ in (80). Assume that the conditions of Lemma 18 and the following condition are satisfied:

(v) For all $x, y, z, w \in C$ one has

$$
\limsup _{t \rightarrow 0^{+}}\langle A z, \eta(x, t y+(1-t) w)\rangle \leq\langle A z, \eta(x, w)\rangle .
$$

Let $\left\{x_{n}\right\}$ be iteratively defined as follows:

$$
\begin{aligned}
& x_{0}=x \in C \quad \text { chosen arbitrarily, } \\
& C_{1, j}=C=C_{0} \\
& y_{n}=\nabla f^{*}\left(\beta_{n} \nabla f\left(x_{n}\right)+\left(1-\beta_{n}\right) \nabla f\left(K_{n} x_{n}\right)\right), \\
& u_{n, j} \in C \quad \text { such that } \\
& g_{j}\left(u_{n, j}, y\right)+\left\langle A_{j} u_{n, j}, \eta\left(y, u_{n, j}\right)\right\rangle+\psi_{j}(y)
\end{aligned}
$$

$$
\begin{aligned}
- & \psi_{j}\left(u_{n, j}\right)+\frac{1}{r_{n}}\left\langle\nabla f\left(u_{n, j}\right)-\nabla f\left(y_{n}\right), y-u_{n, j}\right\rangle \\
\geq 0 & \forall y \in C, \\
C_{n+1, j} & =\left\{z \in C_{n}: D_{f}\left(z, u_{n, j}\right) \leq D_{f}\left(z, x_{n}\right)+\theta_{n}\right\}, \\
C_{n+1} & =\bigcap_{j=1}^{m} C_{n+1, j}, \\
x_{n+1} & =P_{C_{n+1}^{f}}^{f} x_{0}, \quad n \geq 0,
\end{aligned}
$$

where $\left\{\beta_{n}\right\}$ is a real sequence in $(0,1)$ satisfying $\liminf _{n \rightarrow \infty} \beta_{n}\left(1-\beta_{n}\right)>0,\left\{r_{n}\right\} \subset[a, \infty)$ for some $a>0$ and $\theta_{n}=\left(1-\beta_{n}\right) t_{n} \sup _{p \in \mathscr{F}} D_{f}\left(p, x_{n}\right)$. Then $\left\{x_{n}\right\}$ converges strongly to $u=P_{\mathscr{F}}^{f} x_{0}$.

Proof. It follows from Lemmas 20(ii) and 14 that $F\left(K_{n}\right)$ is closed and convex. On the other hand by Lemma 18(5) $\operatorname{EP}\left(g_{j}, A_{j}\right)$ is closed and convex for each $j \in\{1,2,3, \ldots, m\}$; consequently $\mathscr{F}$ is closed and convex.

Next we prove $C_{n}$ is closed and convex. The proof is by induction.

For any fixed $j, C_{1, j}=C$ is closed and convex which implies $C_{1}$ is closed and convex.

Now suppose $C_{k, j}$ is closed and convex for some $k \in \mathbb{N}$. Observe that

$$
\begin{aligned}
& D_{f}\left(z, u_{n, j}\right) \leq D_{f}\left(z, x_{n}\right)+\theta_{n} \Longleftrightarrow \\
& \left\langle\nabla f\left(x_{n}\right)-\nabla f\left(u_{n, j}\right), z\right\rangle \\
& \leq f\left(u_{n, j}\right)-f\left(x_{n}\right)+\left\langle\nabla f\left(u_{n, j}\right)-\nabla f\left(x_{n}\right), u_{n, j}\right\rangle \\
& \quad+\left\langle\nabla f\left(x_{n}\right), x_{n}-u_{n, j}\right\rangle+\theta_{n} .
\end{aligned}
$$

It follows that $C_{k+1, j}$ is closed and convex and so $C_{k+1}$ is closed and convex. Hence $C_{n}$ is closed and convex for all $n \geq 0$. This implies that the iterative sequence is well defined.

Next we show that $\mathscr{F} \subset C_{n} \forall n \geq 0$. Obviously $\mathscr{F} \subset C_{1, j}=$ $C$. Assume $\mathscr{F} \subset C_{k, j}$ for some $k \in \mathbb{N}$ and $\forall j=1,2, \ldots, m$. Let $x^{*} \in \mathscr{F}$; then $x^{*} \in C_{k, j} \forall j=1,2, \ldots, m$ and so $x^{*} \in C_{k}$. Observe from scheme (97) that $u_{n, j}=T_{r_{n}}^{j} y_{n}$. Therefore by Lemma 18(4) we have

$$
D_{f}\left(x^{*}, u_{n, j}\right)=D_{f}\left(x^{*}, T_{r_{n}}^{j} y_{n}\right) \leq D_{f}\left(x^{*}, y_{n}\right) \text {. }
$$

But

$$
\begin{aligned}
D_{f}\left(x^{*}, y_{n}\right) \\
=D_{f}\left(x^{*}, \nabla f^{*}\left(\beta_{n} \nabla f\left(x_{n}\right)+\left(1-\beta_{n}\right) \nabla f\left(K_{n} x_{n}\right)\right)\right) \\
=V_{f}\left(x^{*}, \beta_{n} \nabla f\left(x_{n}\right)+\left(1-\beta_{n}\right) \nabla f\left(K_{n} x_{n}\right)\right) \\
=f\left(x^{*}\right)-\left\langle x^{*}, \beta_{n} \nabla f\left(x_{n}\right)+\left(1-\beta_{n}\right) \nabla f\left(K_{n} x_{n}\right)\right\rangle \\
\quad+f^{*}\left(\beta_{n} \nabla f\left(x_{n}\right)+\left(1-\beta_{n}\right) \nabla f\left(K_{n} x_{n}\right)\right)
\end{aligned}
$$




$$
\begin{aligned}
\leq & f\left(x^{*}\right)-\beta_{n}\left\langle x^{*}, \nabla f\left(x_{n}\right)\right\rangle \\
& -\left(1-\beta_{n}\right)\left\langle x^{*}, \nabla f\left(K_{n} x_{n}\right)\right\rangle+\beta_{n} f^{*}\left(\nabla f\left(x_{n}\right)\right) \\
& +\left(1-\beta_{n}\right) f^{*}\left(\nabla f\left(K_{n} x_{n}\right)\right) \\
= & \beta_{n} V_{f}\left(x^{*}, \nabla f\left(x_{n}\right)\right) \\
& +\left(1-\beta_{n}\right) V_{f}\left(x^{*}, \nabla f\left(K_{n} x_{n}\right)\right) \\
= & \beta_{n} D_{f}\left(x^{*}, x_{n}\right)+\left(1-\beta_{n}\right) D_{f}\left(x^{*}, K_{n} x_{n}\right) \\
\leq & \beta_{n} D_{f}\left(x^{*}, x_{n}\right)+\left(1-\beta_{n}\right)\left(1+t_{n}\right) D_{f}\left(x^{*}, x_{n}\right) \\
= & D_{f}\left(x^{*}, x_{n}\right)+\left(1-\beta_{n}\right) t_{n} D_{f}\left(x^{*}, x_{n}\right) \\
\leq & D_{f}\left(x^{*}, x_{n}\right)+\left(1-\beta_{n}\right) t_{n} \sup _{p \in \mathscr{F}} D_{f}\left(p, x_{n}\right) \\
= & D_{f}\left(x^{*}, x_{n}\right)+\theta_{n} .
\end{aligned}
$$

Thus,

$$
D_{f}\left(x^{*}, u_{n, j}\right) \leq D_{f}\left(x^{*}, x_{n}\right)+\theta_{n} .
$$

This shows that $x^{*} \in C_{k+1, j}$ which implies $\mathscr{F} \subset C_{n, j} \forall n \geq 0$, $\forall j \in\{1,2, \ldots, m\}$. Therefore we obtain $\mathscr{F} \subset C_{n}$.

Next we show that $\lim _{n \rightarrow \infty} D_{f}\left(x_{n}, x_{0}\right)$ exists.

Since $C_{n+1} \subset C_{n} \forall n \geq 0$, we have $x_{n+1}=P_{C_{n+1}} x_{0} \in C_{n+1} \subset$ $C_{n} \forall n \geq 0$. Therefore

$$
D_{f}\left(x_{n}, x_{0}\right)=D_{f}\left(P_{C_{n}}^{f} x_{0}, x_{0}\right) \leq D_{f}\left(x_{n+1}, x_{0}\right),
$$

showing that $\left\{D_{f}\left(x_{n}, x_{0}\right)\right\}$ is nondecreasing sequence of real numbers. On the other hand we have from Lemma 13(ii) that

$$
\begin{aligned}
D_{f}\left(x_{n}, x_{0}\right) & \leq D_{f}\left(x^{*}, x_{0}\right)-D_{f}\left(x^{*}, x_{n}\right) \\
& \leq D_{f}\left(x^{*}, x_{0}\right) .
\end{aligned}
$$

Using (102) and (103) we have that $\lim _{n \rightarrow \infty} D_{f}\left(x_{n}, x_{0}\right)$ exists. Now

$$
\begin{aligned}
D_{f}\left(x_{n+1}, x_{n}\right) & =D_{f}\left(x_{n+1}, P_{C_{n}}^{f} x_{0}\right) \\
& \leq D_{f}\left(x_{n+1}, x_{0}\right)-D_{f}\left(x_{n}, x_{0}\right) .
\end{aligned}
$$

Since $\lim _{n \rightarrow \infty} D_{f}\left(x_{n}, x_{0}\right)$ exists, we obtain

$$
\lim _{n \rightarrow \infty} D_{f}\left(x_{n+1}, x_{n}\right)=0 .
$$

Since $f$ is totally convex on bounded set, by Lemma $7 f$ is sequentially consistent and so we have

$$
\lim _{n \rightarrow \infty}\left\|x_{n+1}-x_{n}\right\|=0 .
$$

Let $m>n$ where $n, m \in \mathbb{N}$. Using (104) we have

$$
\begin{aligned}
D_{f}\left(x_{m}, x_{n}\right) & =D_{f}\left(x_{m}, P_{C_{n}}^{f} x_{0}\right) \\
& \leq D_{f}\left(x_{m}, x_{0}\right)-D_{f}\left(x_{n}, x_{0}\right) .
\end{aligned}
$$

Hence

$$
D_{f}\left(x_{m}, x_{n}\right) \longrightarrow 0 \quad \text { as } n, m \longrightarrow \infty .
$$

It follows that

$$
\left\|x_{m}-x_{n}\right\| \longrightarrow 0 \quad \text { as } n, m \longrightarrow \infty .
$$

This shows that $\left\{x_{n}\right\}$ is Cauchy sequence in $E$. Since $E$ is reflexive and $C$ is weakly closed, then there exists $u \in C$ such that

$$
\lim _{n \rightarrow \infty}\left\|x_{n}-u\right\|=0
$$

Observe that $x_{n+1} \in C_{n+1}=\bigcap_{j=1}^{m} C_{n+1, j}, \forall j \in\{1,2, \ldots, m\}$. Hence we obtain

$$
D_{f}\left(x_{n+1}, u_{n, j}\right) \leq D_{f}\left(x_{n+1}, x_{n}\right)+\theta_{n} .
$$

By (105) and the fact that $\theta_{n} \rightarrow 0$ as $n \rightarrow \infty$, we get

$$
\lim _{n \rightarrow \infty} D_{f}\left(x_{n+1}, u_{n, j}\right)=0 \quad \forall j \in\{1,2, \ldots, m\},
$$

and so

$$
\lim _{n \rightarrow \infty}\left\|x_{n+1}-u_{n, j}\right\|=0, \quad \forall j \in\{1,2, \ldots, m\} .
$$

Now

$$
\left\|u_{n, j}-x_{n}\right\| \leq\left\|u_{n, j}-x_{n+1}\right\|+\left\|x_{n+1}-x_{n}\right\| .
$$

Therefore using (106) and (113) we obtain

$$
\lim _{n \rightarrow \infty}\left\|u_{n, j}-x_{n}\right\|=0, \quad \forall j \in\{1,2, \ldots, m\} .
$$

Also by (110) and (115), it follows that

$$
\lim _{n \rightarrow \infty}\left\|u_{n, j}-u\right\|=0, \quad \forall j \in\{1,2, \ldots, m\} .
$$

Let $r_{2}=\sup \left\{\left\|\nabla f\left(x_{n}\right)\right\|,\left\|\nabla f\left(K_{n} x_{n}\right)\right\|\right\}$. In view of Lemma 9, we have

$$
\begin{aligned}
& D_{f}\left(x^{*}, y_{n}\right) \\
&=D_{f}\left(x^{*}, \nabla f^{*}\left(\beta_{n} \nabla f\left(x_{n}\right)+\left(1-\beta_{n}\right) \nabla f\left(K_{n} x_{n}\right)\right)\right) \\
&=V_{f}\left(x^{*}, \beta_{n} \nabla f\left(x_{n}\right)+\left(1-\beta_{n}\right) \nabla f\left(K_{n} x_{n}\right)\right) \\
&= f\left(x^{*}\right)-\left\langle x^{*}, \beta_{n} \nabla f\left(x_{n}\right)+\left(1-\beta_{n}\right) \nabla f\left(K_{n} x_{n}\right)\right\rangle \\
&+f^{*}\left(\beta_{n} \nabla f\left(x_{n}\right)+\left(1-\beta_{n}\right) \nabla f\left(K_{n} x_{n}\right)\right) \\
& \leq \beta_{n} V_{f}\left(x^{*}, \nabla f\left(x_{n}\right)\right) \\
&+\left(1-\beta_{n}\right) V_{f}\left(x^{*}, \nabla f\left(K_{n} x_{n}\right)\right) \\
&-\beta_{n}\left(1-\beta_{n}\right) \rho_{r_{2}}^{*}\left(\left\|\nabla f\left(x_{n}\right)-\nabla f\left(K_{n} x_{n}\right)\right\|\right) \\
&= \beta_{n} D_{f}\left(x^{*}, x_{n}\right)+\left(1-\beta_{n}\right) D_{f}\left(x^{*}, K_{n} x_{n}\right) \\
&-\beta_{n}\left(1-\beta_{n}\right) \rho_{r_{2}}^{*}\left(\left\|\nabla f\left(x_{n}\right)-\nabla f\left(K_{n} x_{n}\right)\right\|\right) .
\end{aligned}
$$


From (99), we have

$$
\begin{aligned}
D_{f} & \left(x^{*}, u_{n, j}\right) \\
& \leq \beta_{n} D_{f}\left(x^{*}, x_{n}\right)+\left(1-\beta_{n}\right) D_{f}\left(x^{*}, K_{n} x_{n}\right) \\
& \quad-\beta_{n}\left(1-\beta_{n}\right) \rho_{r_{2}}^{*}\left(\left\|\nabla f\left(x_{n}\right)-\nabla f\left(K_{n} x_{n}\right)\right\|\right) .
\end{aligned}
$$

Therefore by Lemma 20(i), we have

$$
\begin{aligned}
& D_{f}\left(x^{*}, u_{n, j}\right) \\
& \leq \beta_{n} D_{f}\left(x^{*}, x_{n}\right)+\left(1-\beta_{n}\right)\left(1+t_{n}\right) D_{f}\left(x^{*}, x_{n}\right) \\
&-\beta_{n}\left(1-\beta_{n}\right) \rho_{r_{2}}^{*}\left(\left\|\nabla f\left(x_{n}\right)-\nabla f\left(K_{n} x_{n}\right)\right\|\right) \\
&= D_{f}\left(x^{*}, x_{n}\right)+\left(1-\beta_{n}\right) t_{n} D_{f}\left(x^{*}, x_{n}\right) \\
&-\beta_{n}\left(1-\beta_{n}\right) \rho_{r_{2}}^{*}\left(\left\|\nabla f\left(x_{n}\right)-\nabla f\left(K_{n} x_{n}\right)\right\|\right) \\
& \leq D_{f}\left(x^{*}, x_{n}\right)+\left(1-\beta_{n}\right) t_{n} \sup _{p \in \mathscr{F}} D_{f}\left(p, x_{n}\right) \\
&-\beta_{n}\left(1-\beta_{n}\right) \rho_{r_{2}}^{*}\left(\left\|\nabla f\left(x_{n}\right)-\nabla f\left(K_{n} x_{n}\right)\right\|\right) \\
&= D_{f}\left(x^{*}, x_{n}\right)+\theta_{n} \\
&-\beta_{n}\left(1-\beta_{n}\right) \rho_{r_{2}}^{*}\left(\left\|\nabla f\left(x_{n}\right)-\nabla f\left(K_{n} x_{n}\right)\right\|\right) .
\end{aligned}
$$

Observe that

$$
\begin{aligned}
& D_{f}\left(x^{*}, x_{n}\right)-D_{f}\left(x^{*}, u_{n, j}\right) \\
&= f\left(u_{n, j}\right)-f\left(x_{n}\right)+\left\langle\nabla f\left(u_{n, j}\right), x^{*}-u_{n, j}\right\rangle \\
&-\left\langle\nabla f\left(x_{n}\right), x^{*}-x_{n}\right\rangle \\
&= f\left(u_{n, j}\right)-f\left(x_{n}\right)+\left\langle\nabla f\left(u_{n, j}\right), x^{*}-x_{n}\right\rangle \\
&+\left\langle\nabla f\left(u_{n, j}\right), x_{n}-u_{n, j}\right\rangle-\left\langle\nabla f\left(x_{n}\right), x^{*}-x_{n}\right\rangle \\
&= f\left(u_{n, j}\right)-f\left(x_{n}\right) \\
&+\left\langle\nabla f\left(u_{n, j}\right)-\nabla f\left(x_{n}\right), x^{*}-x_{n}\right\rangle \\
&+\left\langle\nabla f\left(u_{n, j}\right), x_{n}-u_{n, j}\right\rangle .
\end{aligned}
$$

Therefore

$$
\begin{aligned}
& \left|D_{f}\left(x^{*}, x_{n}\right)-D_{f}\left(x^{*}, u_{n, j}\right)\right| \\
& \leq\left|f\left(u_{n, j}\right)-f\left(x_{n}\right)\right| \\
& \quad+\left|\left\langle\nabla f\left(u_{n, j}\right)-\nabla f\left(x_{n}\right), x^{*}-x_{n}\right\rangle\right| \\
& \quad+\left|\left\langle\nabla f\left(u_{n, j}\right), x_{n}-u_{n, j}\right\rangle\right| \\
& \leq\left|f\left(u_{n, j}\right)-f\left(x_{n}\right)\right| \\
& \quad+\left\|\nabla f\left(u_{n, j}\right)-\nabla f\left(x_{n}\right)\right\|\left\|x^{*}-x_{n}\right\| \\
& \quad+\left\|f\left(u_{n, j}\right)\right\|\left\|x_{n}-u_{n, j}\right\| .
\end{aligned}
$$

From (115), we obtain

$$
\begin{aligned}
&\left|D_{f}\left(x^{*}, x_{n}\right)-D_{f}\left(x^{*}, u_{n, j}\right)\right| \longrightarrow 0 \\
& \text { as } n \longrightarrow \infty \forall j \in\{1,2, \ldots, m\} .
\end{aligned}
$$

By (122) and $\lim \inf _{n \rightarrow \infty} \beta_{n}\left(1-\beta_{n}\right)>0$, it follows from (119) that

$$
\lim _{n \rightarrow \infty} \rho_{r_{2}}^{*}\left(\left\|\nabla f\left(x_{n}\right)-\nabla f\left(K_{n} x_{n}\right)\right\|\right)=0
$$

From the property of $\rho_{r_{2}}^{*}$, we deduce $\lim _{n \rightarrow \infty} \| \nabla f\left(x_{n}\right)-$ $\nabla f\left(K_{n} x_{n}\right) \|=0$. By uniform continuity of $\nabla f^{*}$ on bounded subsets of $E$, we obtain

$$
\lim _{n \rightarrow \infty}\left\|x_{n}-K_{n} x_{n}\right\|=0
$$

On the other hand

$$
\left\|K_{n} x_{n}-u\right\| \leq\left\|K_{n} x_{n}-x_{n}\right\|+\left\|x_{n}-u\right\| .
$$

From (110) and (124), we have

$$
\lim _{n \rightarrow \infty}\left\|K_{n} x_{n}-u\right\|=0 .
$$

Now using (110), (126), and Lemma 20(iii), we obtain $K_{n} u=$ $u \forall n \in \mathbb{N}$. This implies $u \in F\left(K_{n}\right)=\bigcap_{i=1}^{N} F\left(T_{i}\right)$.

Next we show $u \in \operatorname{EP}\left(g_{j}, A_{j}\right)$.

From scheme (97)

$$
\begin{aligned}
\left\|\nabla f\left(x_{n}\right)-\nabla f\left(y_{n}\right)\right\| \\
\quad=\left(1-\beta_{n}\right)\left\|\nabla f\left(x_{n}\right)-\nabla f\left(K_{n} x_{n}\right)\right\| .
\end{aligned}
$$

Therefore by (124) and uniform continuity of $\nabla f$, we have

$$
\lim _{n \rightarrow \infty}\left\|\nabla f\left(x_{n}\right)-\nabla f\left(y_{n}\right)\right\|=0 .
$$

As $\nabla f^{*}$ is uniformly continuous, we obtain

$$
\lim _{n \rightarrow \infty}\left\|x_{n}-y_{n}\right\|=0
$$

From (115) and (129), we have

$$
\lim _{n \rightarrow \infty}\left\|u_{n, j}-y_{n}\right\|=0 \quad \forall j=1,2, \ldots, m .
$$

Again since $\nabla f$ is uniformly continuous and $r_{n}>a$, we get

$$
\lim _{n \rightarrow \infty} \frac{\left\|\nabla f\left(u_{n, j}\right)-\nabla f\left(y_{n}\right)\right\|}{r_{n}}=0 \quad \forall j=1,2, \ldots, m .
$$

From scheme (97)

$$
\begin{aligned}
& g_{j}\left(u_{n, j}, y\right)+\left\langle A_{j} u_{n, j}, \eta\left(y, u_{n, j}\right)\right\rangle+\psi_{j}(y) \\
& -\psi_{j}\left(u_{n, j}\right) \\
& +\frac{1}{r_{n}}\left\langle\nabla f\left(u_{n, j}\right)-\nabla f\left(y_{n}\right), y-u_{n, j}\right\rangle \geq 0
\end{aligned}
$$


Using (C2) and Lemma 17(ii), it follows that

$$
\begin{aligned}
\frac{1}{r_{n}}\left\|\nabla f\left(u_{n, j}\right)-\nabla f\left(y_{n}\right)\right\|\left\|u_{n, j}-y\right\| \\
\geq\left\langle A_{j} u_{n, j}, \eta\left(u_{n, j}, y\right)\right\rangle+\psi_{j}\left(u_{n, j}\right)-\psi_{j}(y) \\
\quad-g_{j}\left(u_{n, j}, y\right) \quad \forall y \in C \\
\geq\left\langle A_{j} u_{n, j}, \eta\left(u_{n, j}, y\right)\right\rangle+\psi_{j}\left(u_{n, j}\right)-\psi_{j}(y) \\
\quad+g_{j}\left(y, u_{n, j}\right) \quad \forall y \in C .
\end{aligned}
$$

Using (116) and (131) and taking $\lim \inf$ as $n \rightarrow \infty$ of the above inequality, we get

$$
\begin{array}{r}
0 \geq\left\langle A_{j} u, \eta(u, y)\right\rangle+\psi_{j}(u)-\psi_{j}(y)+g_{j}(y, u) \\
\forall y \in C \forall j=1,2, \ldots, m .
\end{array}
$$

Now for any $t \in(0,1)$ and $y \in C$, let $y_{t}=t y+(1-t) u$. Then $y_{t} \in C$ and so

$$
\begin{array}{r}
0 \geq\left\langle A_{j} u, \eta\left(u, y_{t}\right)\right\rangle+\psi_{j}(u)-\psi_{j}\left(y_{t}\right)+g_{j}\left(y_{t}, u\right) \\
\forall j=1,2, \ldots, m .
\end{array}
$$

Therefore by (C1), (C4), Lemma 18(i), (ii), and (135), we have

$$
\begin{aligned}
0= & g_{j}\left(y_{t}, y_{t}\right)+\left\langle A_{j} u, \eta\left(y_{t}, y_{t}\right)\right\rangle+\psi_{j}\left(y_{t}\right) \\
& -\psi_{j}\left(y_{t}\right)=g_{j}\left(y_{t}, t y+(1-t) u\right) \\
& +\left\langle A_{j} u, \eta\left(t y+(1-t) u, y_{t}\right)\right\rangle+\psi_{j}(t y \\
& +(1-t) u)-\psi_{j}\left(y_{t}\right) \leq t\left[g_{j}\left(y_{t}, y\right)\right. \\
& \left.+\left\langle A_{j} u, \eta\left(y, y_{t}\right)\right\rangle+\psi_{j}(y)-\psi_{j}\left(y_{t}\right)\right]+(1-t) \\
& +\left[g_{j}\left(y_{t}, u\right)+\left\langle A_{j} u, \eta\left(u, y_{t}\right)\right\rangle+\psi_{j}(u)\right. \\
& \left.-\psi_{j}\left(y_{t}\right)\right] \leq t\left[g_{j}\left(y_{t}, y\right)+\left\langle A_{j} u, \eta\left(y, y_{t}\right)\right\rangle\right. \\
& \left.+\psi_{j}(y)-\psi_{j}\left(y_{t}\right)\right] .
\end{aligned}
$$

That is,

$$
g_{j}\left(y_{t}, y\right)+\left\langle A_{j} u, \eta\left(y, y_{t}\right)\right\rangle+\psi_{j}(y)-\psi_{j}\left(y_{t}\right) \geq 0 \text {. }
$$

Since $y_{t}=t y+(1-t) u$, we have

$$
\begin{gathered}
g_{j}(t y+(1-t) u, y)+\left\langle A_{j} u, \eta(y, t y+(1-t) u)\right\rangle \\
+\psi_{j}(y)-\psi_{j}(t y+(1-t) u) \geq 0 .
\end{gathered}
$$

From (C3), (v), and lower semicontinuity of $\psi$, we have by allowing $t \rightarrow 0^{+}$

$$
\begin{aligned}
& g_{j}(u, y)+\left\langle A_{j} u, \eta(y, u)\right\rangle+\psi_{j}(y)-\psi_{j}(u) \geq 0 \\
& \forall y \in C .
\end{aligned}
$$

This shows that $u \in \operatorname{EP}\left(g_{j}, A_{j}\right)$.

Lastly we show $u=P_{\mathscr{F}}^{f} x_{0}$.

From $x_{n}=P_{C_{n}}^{f} x_{0}$ and Lemma 13(i) we have

$$
\left\langle\nabla f\left(x_{0}\right)-\nabla f\left(x_{n}\right), x_{n}-z\right\rangle \geq 0 \quad \forall z \in C_{n} .
$$

Since $\mathscr{F} \subset C_{n}$, this implies that

$$
\left\langle\nabla f\left(x_{0}\right)-\nabla f\left(x_{n}\right), x_{n}-h\right\rangle \geq 0 \quad \forall h \in \mathscr{F} .
$$

Letting $n \rightarrow \infty$ in (141), we obtain

$$
\left\langle\nabla f\left(x_{0}\right)-\nabla f\left(x_{n}\right), x_{n}-h\right\rangle \geq 0 \quad \forall h \in \mathscr{F} .
$$

Again by Lemma 13(i) we have $u=P_{\mathscr{F}}^{f} x_{0}$. This completes the proof.

If $N=1$ and $m=1$, in Theorem 21 then we have the following corollary.

Corollary 22. Let $E$ be a reflexive Banach space with the dual $E^{*}$ and let $C$ be a nonempty, closed, convex, and bounded subset of $E$. Let $f: E \rightarrow(-\infty,+\infty]$ be a strongly coercive, Legendre, uniformly Fréchet differentiable, and totally convex function which is bounded on bounded subsets of E. Let $A: C \rightarrow E^{*}$ be $\eta$-hemicontinuous and relaxed $\eta-\alpha$ monotone mapping and $g$ : $C \times C \rightarrow \mathbb{R}$ be bifunctions satisfying $(C 1)-(C 4)$. Let $\psi: C \rightarrow \mathbb{R}$ be a proper, convex, and lower semicontinuous mapping. Let $T$ be a continuous Bregman quasiasymptotically nonexpansive mapping of $C$ into itself such that $\mathscr{F}=F(T) \cap E P(g, A) \neq \emptyset$. Assume that the conditions of Lemma 18 and the following condition are satisfied:

(v) For all $x, y, z, w \in C$ one has

$$
\limsup _{t \rightarrow 0^{+}}\langle A z, \eta(x, t y+(1-t) w)\rangle \leq\langle A z, \eta(x, w)\rangle .
$$

Let $\left\{x_{n}\right\}$ be iteratively defined as follows:

$x_{0}=x \in C$ chosen arbitrarily,

$y_{n}=\nabla f^{*}\left(\beta_{n} \nabla f\left(x_{n}\right)+\left(1-\beta_{n}\right) \nabla f\left(T^{n} x_{n}\right)\right)$,

$u_{n} \in C$ such that

$$
\begin{aligned}
& g\left(u_{n}, y\right)+\left\langle A u_{n}, \eta\left(y, u_{n}\right)\right\rangle+\psi(y)-\psi\left(u_{n}\right) \\
& \quad+\frac{1}{r_{n}}\left\langle\nabla f\left(u_{n}\right)-\nabla f\left(y_{n}\right), y-u_{n}\right\rangle \geq 0 \quad \forall y \in C, \\
& C_{n+1}=\left\{z \in C_{n}: D_{f}\left(z, u_{n}\right) \leq D_{f}\left(z, x_{n}\right)+\theta_{n}\right\}, \\
& x_{n+1}=P_{C_{n+1}}^{f} x_{0}, \quad n \geq 0,
\end{aligned}
$$


where $\left\{\beta_{n}\right\}$ is a real sequence in $(0,1)$ satisfying $\liminf \operatorname{in}_{n \rightarrow \infty} \beta_{n}\left(1-\beta_{n}\right)>0,\left\{r_{n}\right\} \subset[a, \infty)$ for some $a>0$ and $\theta_{n}=\left(1-\beta_{n}\right) t_{n} \sup _{p \in \mathscr{F}} D_{f}\left(p, x_{n}\right)$. Then $\left\{x_{n}\right\}$ converges strongly to $u=P_{\mathscr{F}}^{f} x_{0}$.

Setting $A_{j}=0 \forall j=1,2, \ldots, m$ in Theorem 21 then we obtain the following result.

Corollary 23. Let $E$ be a reflexive Banach space with the dual $E^{*}$ and let $C$ be a nonempty, closed, convex, and bounded subset of $E$. Let $f: E \rightarrow(-\infty,+\infty]$ be a strongly coercive, Legendre, uniformly Fréchet differentiable, and totally convex function which is bounded on bounded subsets of $E$. For each $j=1,2,3, \ldots, m$, let $g_{j}: C \times C \rightarrow \mathbb{R}$ be bifunctions satisfying $(C 1)-(C 4)$ and $\psi_{j}: C \rightarrow \mathbb{R}$ be proper, convex, and lower semicontinuous mappings. Let $\left\{T_{i}\right\}_{i=1}^{N}$ be a finite family of continuous Bregman quasiasymptotically nonexpansive mapping of $C$ into itself such that $\mathscr{F}=\left[\bigcap_{i=1}^{N} F\left(T_{i}\right)\right] \cap$ $\left[E P\left(g_{j}, \psi_{j}\right)\right] \neq \emptyset$. Let $K_{n}$ be the Bregman K-mapping generated by $T_{1}, T_{2}, T_{3}, \ldots, T_{N}$ in (80). Let $\left\{x_{n}\right\}$ be iteratively defined as follows:

$$
\begin{aligned}
& x_{0}=x \in C \quad \text { chosen arbitrarily, } \\
& C_{1, j}=C=C_{0} \\
& y_{n}=\nabla f^{*}\left(\beta_{n} \nabla f\left(x_{n}\right)+\left(1-\beta_{n}\right) \nabla f\left(K_{n} x_{n}\right)\right), \\
& u_{n, j} \in C \quad \text { such that } \\
& g_{j}\left(u_{n, j}, y\right)+\psi_{j}(y)-\psi_{j}\left(u_{n, j}\right) \\
& \quad+\frac{1}{r_{n}}\left\langle\nabla f\left(u_{n, j}\right)-\nabla f\left(y_{n}\right), y-u_{n, j}\right\rangle \geq 0 \\
& C_{n+1, j}=\left\{z \in C_{n}: D_{f}\left(z, u_{n, j}\right) \leq D_{f}\left(z, x_{n}\right)+\theta_{n}\right\}, \\
& C_{n+1}=\bigcap_{j=1}^{m} C_{n+1, j}, \\
& x_{n+1}=P_{C_{n+1}}^{f} x_{0}, \quad n \geq 0,
\end{aligned}
$$

where $\left\{\beta_{n}\right\}$ is a real sequence in $(0,1)$ satisfying $\liminf \operatorname{in}_{n \rightarrow \infty} \beta_{n}\left(1-\beta_{n}\right)>0,\left\{r_{n}\right\} \subset[a, \infty)$ for some $a>0$ and $\theta_{n}=\left(1-\beta_{n}\right) t_{n} \sup _{p \in \mathscr{F}} D_{f}\left(p, x_{n}\right)$. Then $\left\{x_{n}\right\}$ converges strongly to $u=P_{\mathscr{F}}^{f} x_{0}$.

If $A_{j}=0$ and $\psi_{j}=0 \forall j=1,2, \ldots, m$ in Theorem 21 then we obtain the following result.

Corollary 24. Let $E$ be a reflexive Banach space with the dual $E^{*}$ and let $C$ be a nonempty, closed, convex, and bounded subset of $E$. Let $f: E \rightarrow(-\infty,+\infty]$ be a strongly coercive, Legendre, uniformly Fréchet differentiable, and totally convex function which is bounded on bounded subsets of $E$. For each $j=1,2,3, \ldots, m$, let $g_{j}: C \times C \rightarrow \mathbb{R}$ be bifunctions satisfying (C1)-(C4). Let $\left\{T_{i}\right\}_{i=1}^{N}$ be a finite family of continuous Bregman quasiasymptotically nonexpansive mappings of $C$ into itself such that $\mathscr{F}=\left[\bigcap_{i=1}^{N} F\left(T_{i}\right)\right] \cap\left[E P\left(g_{j}\right)\right] \neq \emptyset$. Let $K_{n}$ be the Bregman K-mapping generated by $T_{1}, T_{2}, T_{3}, \ldots, T_{N}$ in (80). Let $\left\{x_{n}\right\}$ be iteratively defined as follows:

$$
\begin{aligned}
& x_{0}=x \in C \text { chosen arbitrarily, } \\
& C_{1, j}=C=C_{0} \\
& y_{n}=\nabla f^{*}\left(\beta_{n} \nabla f\left(x_{n}\right)+\left(1-\beta_{n}\right) \nabla f\left(K_{n} x_{n}\right)\right), \\
& u_{n, j} \in C \quad \text { such that } \\
& g_{j}\left(u_{n, j}, y\right)+\frac{1}{r_{n}}\left\langle\nabla f\left(u_{n, j}\right)-\nabla f\left(y_{n}\right), y-u_{n, j}\right\rangle \geq 0 \\
& C_{n+1, j}=\left\{z \in C_{n}: D_{f}\left(z, u_{n, j}\right) \leq D_{f}\left(z, x_{n}\right)+\theta_{n}\right\}, \\
& C_{n+1}=\bigcap_{j=1}^{m} C_{n+1, j}, \\
& x_{n+1}=P_{C_{n+1}}^{f} x_{0}, \quad n \geq 0,
\end{aligned}
$$

where $\left\{\beta_{n}\right\}$ is a real sequence in $(0,1)$ satisfying $\liminf _{n \rightarrow \infty} \beta_{n}\left(1-\beta_{n}\right)>0,\left\{r_{n}\right\} \subset[a, \infty)$ for some $a>0$ and $\theta_{n}=\left(1-\beta_{n}\right) t_{n} \sup _{p \in \mathscr{F}} D_{f}\left(p, x_{n}\right)$. Then $\left\{x_{n}\right\}$ converges strongly to $u=P_{\mathscr{F}}^{f} x_{0}$.

As a direct consequence of Theorem 21 and Remark 1, we obtain the convergence result for system of the mixed equilibrium problems and finite family of quasi- $\phi$-asymptotically nonexpansive mappings in uniformly convex and uniformly smooth Banach spaces.

Corollary 25. Let $E$ be a uniformly convex and uniformly smooth Banach space with the dual $E^{*}$ and let $C$ be a nonempty, closed, convex, and bounded subset of E. Let $J$ : $E \rightarrow E^{*}$ be a normalised duality mapping. For each $j=$ $1,2,3, \ldots, m$, let $A_{j}: C \rightarrow E^{*}$ be $\eta$-hemicontinuous and relaxed $\eta-\alpha$ monotone mappings and $g_{j}: C \times C \rightarrow \mathbb{R}$ be bifunctions satisfying $(C 1)-(C 4)$. Let $\psi_{j}: C \rightarrow \mathbb{R}$ be proper, convex, and lower semicontinuous mappings. Let $\left\{T_{i}\right\}_{i=1}^{N}$ be a finite family of continuous Bregman quasiasymptotically nonexpansive mappings of $C$ into itself such that $\mathscr{F}=$ $\left[\cap_{i=1}^{N} F\left(T_{i}\right)\right] \cap\left[E P\left(g_{j}, A_{j}\right)\right] \neq \emptyset$. Let $K_{n}$ be the Bregman $K-$ mapping generated by $T_{1}, T_{2}, T_{3}, \ldots, T_{N}$ in (80). Assume that the conditions of Lemma 18 and the following condition are satisfied:

(v) For all $x, y, z, w \in C$ one has

$$
\limsup _{t \rightarrow 0^{+}}\langle A z, \eta(x, t y+(1-t) w)\rangle \leq\langle A z, \eta(x, w)\rangle .
$$


Let $\left\{x_{n}\right\}$ be iteratively defined as follows:

$x_{0}=x \in C$ chosen arbitrarily,

$C_{1, j}=C=C_{0}$

$y_{n}=J^{-1}\left(\beta_{n} J\left(x_{n}\right)+\left(1-\beta_{n}\right) J\left(K_{n} x_{n}\right)\right)$,

$u_{n, j} \in C$ such that

$g_{j}\left(u_{n, j}, y\right)+\left\langle A_{j} u_{n, j}, \eta\left(y, u_{n, j}\right)\right\rangle+\psi_{j}(y)$

$-\psi_{j}\left(u_{n, j}\right)+\frac{1}{r_{n}}\left\langle J\left(u_{n, j}\right)-J\left(y_{n}\right), y-u_{n, j}\right\rangle \geq 0$

$\forall y \in C$,

$C_{n+1, j}=\left\{z \in C_{n}: \phi\left(z, u_{n, j}\right) \leq \phi\left(z, x_{n}\right)+\theta_{n}\right\}$,

$C_{n+1}=\bigcap_{j=1}^{m} C_{n+1, j}$,

$x_{n+1}=\Pi_{C_{n+1}} x_{0}, \quad n \geq 0$,

where $\left\{\beta_{n}\right\}$ is a real sequence in $(0,1)$ satisfying $\liminf _{n \rightarrow \infty} \beta_{n}\left(1-\beta_{n}\right)>0,\left\{r_{n}\right\} \subset[a, \infty)$ for some $a>0$ and $\theta_{n}=\left(1-\beta_{n}\right) t_{n} \sup _{p \in \mathscr{F}} D_{f}\left(p, x_{n}\right)$. Then $\left\{x_{n}\right\}$ converges strongly to $u=P_{\mathscr{F}}^{f} x_{0}$.

Remark 26. Our theorems and corollaries generalise the main theorem of Chen et al. [27] in the following senses:

(1) For the structure of Banach spaces, we extend the duality mapping to more general case: that is, a Legendre, strongly coercive, uniformly Fréchet differentiable, and totally convex function.

(2) For the mapping, we consider Bregman quasiasymptotically nonexpansive mappings which contain Bregman quasi nonexpansive mappings as a special case which itself is generalisation of quasi- $\phi$-nonexpansive mappings.

(3) In Chen et al. [27] the authors considered mixed equilibrium problems while in this paper a system of equilibrium problems is considered.

\section{Competing Interests}

The authors declare that they have no competing interests.

\section{References}

[1] E. Blum and W. Oettli, "From optimization and variational inequalities to equilibrium problems," The Mathematics Student, vol. 63, no. 1-4, pp. 123-145, 1994.

[2] Y. P. Fang and N. J. Huang, "Variational-like inequalities with generalized monotone mappings in Banach spaces," Journal of Optimization Theory and Applications, vol. 118, no. 2, pp. 327338, 2003.

[3] A. H. Siddiqi, Q. H. Ansari, and K. R. Kazmi, "On nonlinear variational inequalities," Indian Journal of Pure and Applied Mathematics, vol. 25, no. 9, pp. 969-973, 1994.
[4] D. Goeleven and D. Motreanu, "Eigenvalue and dynamic problems for variational and hemivariational inequalities," Communications on Applied Nonlinear Analysis, vol. 3, no. 4, pp. $1-21,1996$.

[5] R. U. Verma, "Nonlinear variational inequalities on convex subsets of Banach spaces," Applied Mathematics Letters, vol. 10, no. 4, pp. 25-27, 1997.

[6] L.-C. Ceng and J.-C. Yao, "A hybrid iterative scheme for mixed equilibrium problems and fixed point problems," Journal of Computational and Applied Mathematics, vol. 214, no. 1, pp. 186201, 2008.

[7] S. H. Wang, G. Marino, and F. H. Wang, "Strong convergence theorems for a generalized equilibrium problem with a relaxed monotone mapping and a countable family of nonexpansive mappings in a Hilbert space," Fixed Point Theory and Applications, vol. 2010, Article ID 230304, 2010.

[8] B. Ali, "Fixed points approximation and solutions of some equilibrium and variational inequalities problems," International Journal of Mathematics and Mathematical Sciences, vol. 2009, Article ID 656534, 17 pages, 2009.

[9] P. Daniele, F. Giannessi, and A. Maugeri, Equilibrium Problems and Variational Models, Kluwer Academic, Dordrecht, The Netherlands, 2003.

[10] N. Petrot, K. Wattanawitoon, and P. Kumam, "A hybrid projection method for generalized mixed equilibrium problems and fixed point problems in Banach spaces," Nonlinear Analysis: Hybrid Systems, vol. 4, no. 4, pp. 631-643, 2010.

[11] W. Takahashi and K. Zembayash, "Strong convergence theorem by a new hybrid method for equilibrium problems and relatively nonexpansive mappings," Fixed Point Theory and Applications, vol. 2008, Article ID 528476, 11 pages, 2008.

[12] S. Wang and B. Guo, "New iterative scheme with nonexpansive mappings for equilibrium problems and variational inequality problems in Hilbert spaces," Journal of Computational and Applied Mathematics, vol. 233, no. 10, pp. 2620-2630, 2010.

[13] K. Wattanawitoon and P. Kumam, "Generalized mixed equilibrium problems for maximal monotone operators and two relatively quasi-nonexpansive mappings," Thai Journal of Mathematics, vol. 9, no. 1, pp. 165-189, 2011.

[14] Q. Yuan and M. J. Shang, "Ky Fan inequalities, $\phi$-distances, and generalizesd asymptotically quasi- $\phi$-nonexpansive mappings," Journal Fixed Point Theory, vol. 8, 2013.

[15] Q. N. Zhang and H. L. Wu, "Hybrid algorithms for equilibrium and common fixed point problems with applications," Journal of Inequalities and Applications, vol. 2014, article 221, 2014.

[16] H. H. Bauschke, J. M. Borwein, and P. L. Combettes, "Essential smoothness, essential strict convexity, and Legendre functions in Banach spaces," Communications in Contemporary Mathematics, vol. 3, no. 4, pp. 615-647, 2001.

[17] W. Takahashi, Convex Analysis and Approximation of Fixed Points, Yokahama Publishers, Yokahama, Japan, 2000.

[18] A. Ambrosetti and G. Prodi, A Primer of Nonlinear Analysis, vol. 34 of Cambridge Studies in Advanced Mathematics, Cambridge University Press, Cambridge, UK, 1993.

[19] R. R. Phelps, Convex Functions, Monotone Operators and Differentiability, vol. 1364 of Lecture Notes in Mathematics, Springer, Berlin, Germany, 2nd edition, 1993.

[20] J. F. Bonnans and A. Shapiro, Perturbation Analysis of Optimization Problems, Springer, New York, NY, USA, 2000.

[21] G. C. Ugwunnadi, B. Ali, I. Idris, and M. S. Minjibir, "Strong convergence theorem for quasi-Bregman strictly pseudocontractive mappings and equilibrium problems in BANach 
spaces," Fixed Point Theory and Applications, vol. 2014, article 231, 16 pages, 2014.

[22] L. M. Bregman, "The relation method of finding the common points of convex sets and its application to solution of problems in convex programming, USSR," Computational Mathematics and Mathematical Physics, vol. 7, pp. 200-217, 1967.

[23] Y. Censor and A. Lent, "An iterative row-action method for interval convex programming," Journal of Optimization Theory and Applications, vol. 34, no. 3, pp. 321-353, 1981.

[24] D. Butnariu and A. N. Lusem, Totally Convex Functions for Fixed Point Computation and Finite Dimensional Optimization, Kluwer Academic, Dordrecht, The Netherlands, 2012.

[25] Y. I. Alber, "Metric and generalized projection operators in Banach spaces: properties and applications," in Theory and Applications of Nonlinear Operators of Accretive and Monotone Type, vol. 178 of Lecture Notes in Pure and Applied Mathematics, pp. 15-50, 1996.

[26] V. Martín-Márquez, S. Reich, and S. Sabach, "Iterative methods for approximating fixed points of Bregman nonexpansive operators," Discrete and Continuous Dynamical Systems. Series S, vol. 6, no. 4, pp. 1043-1063, 2013.

[27] M. Chen, J. Song, and S. Wang, "New mixed equilibrium problems and iterative algorithms for fixed point problems in Banach spaces," Journal of Applied Mathematics, vol. 2014, Article ID 193749, 10 pages, 2014.

[28] C. Zălinescu, Convex Analysis in General Vector Spaces, World Scientific Publishing, River Edge, NJ, USA, 2002.

[29] D. Butnariu and E. Resmerita, "Bregman distances, totally convex functions, and a method for solving operator equations in Banach spaces," Abstract and Applied Analysis, vol. 2006, Article ID 84919, 39 pages, 2006.

[30] K. K. Marzukiewicz, "Ein beweis des fixpuntsatzen fur ndimensionale simplexe," Fundamenta Mathematicae, vol. 14, pp. 132-137, 1929.

[31] K. Fan, "Some properties of convex sets related to fixed point theorems," Mathematische Annalen, vol. 266, no. 4, pp. 519-537, 1984.

[32] E. Resmerita, "On total convexity, Bregman projections and stability in BANach spaces," Journal of Convex Analysis, vol. 11, no. 1, pp. 1-16, 2004.

[33] E. Naraghirad and J.-C. Yao, "Bregman weak relatively nonexpansive mappings in Banach spaces," Fixed Point Theory and Applications, vol. 2013, article 141, 2013.

[34] S. Reich and S. Sabach, "A strong convergence theorem for a proximal-type algorithm in reflexive Banach spaces," Journal of Nonlinear and Convex Analysis, vol. 10, no. 3, pp. 471-485, 2009.

[35] Y. H. Zhao, S. S. Chang, and J. H. Zhu, "Strong convergence theorems for Bregman quasi-asymptotically nonexpansive mappings and equilibrium problem in reflexive Banach spaces," Mathematical Inequalities \& Applications, vol. 16, no. 4, pp. 11711183, 2013. 


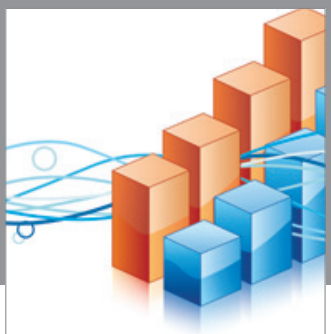

Advances in

Operations Research

vatem alat4

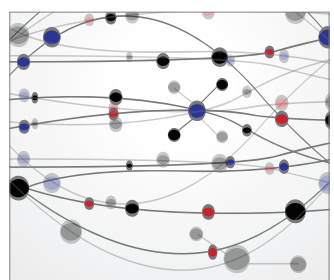

\section{The Scientific} World Journal
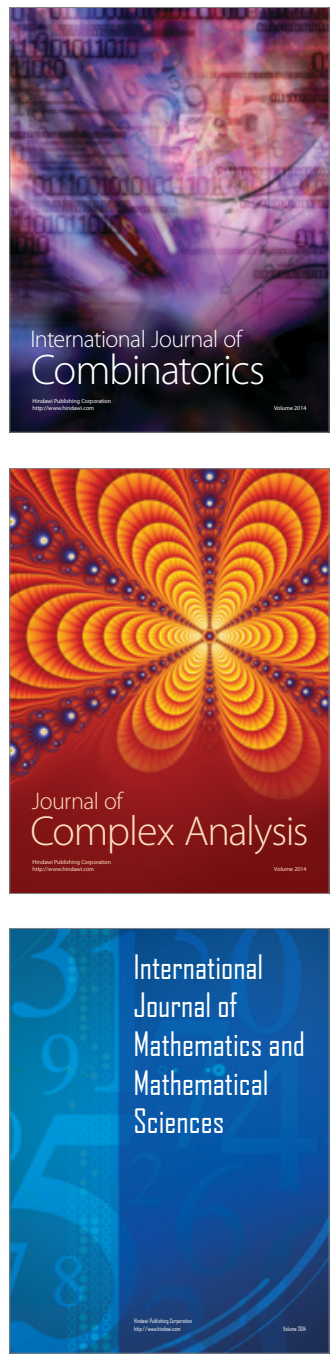
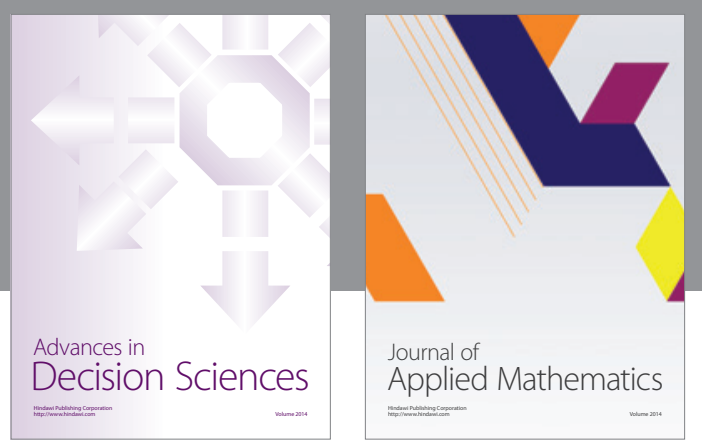

Algebra

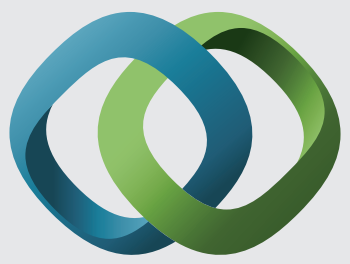

\section{Hindawi}

Submit your manuscripts at

http://www.hindawi.com
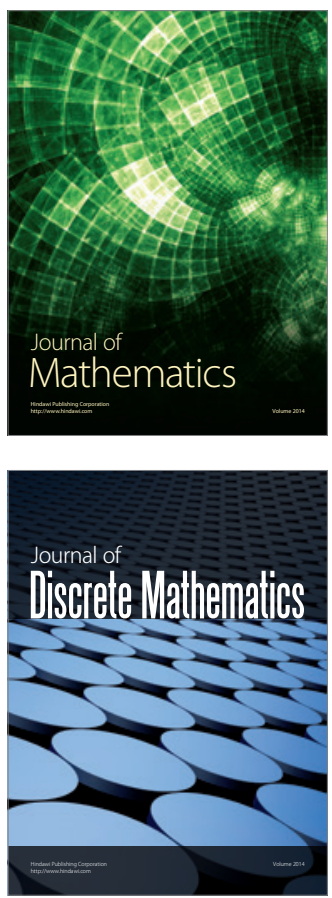

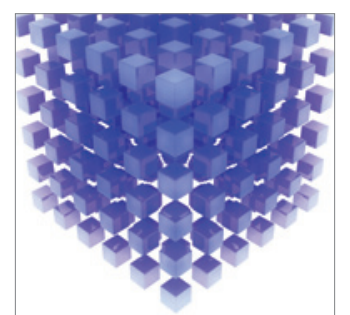

Mathematical Problems in Engineering
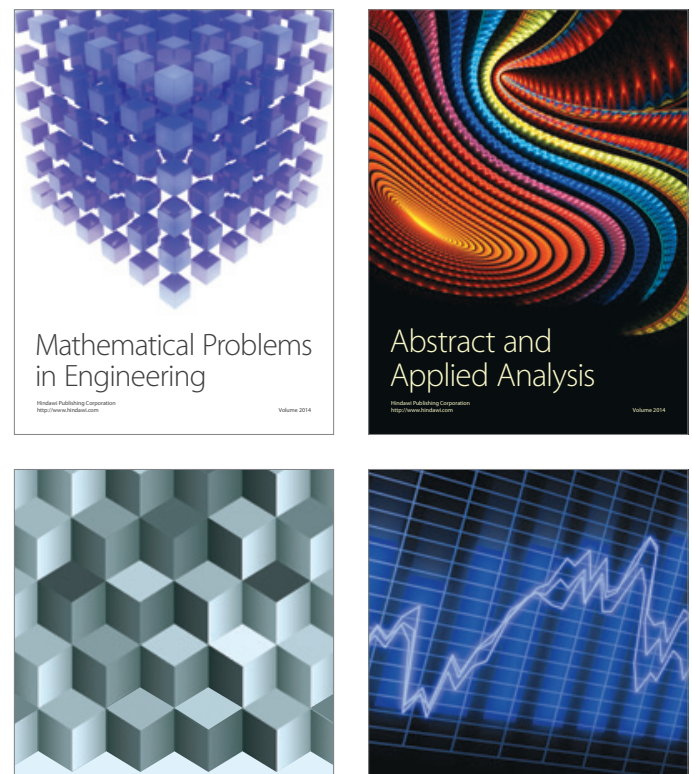

Journal of

Function Spaces

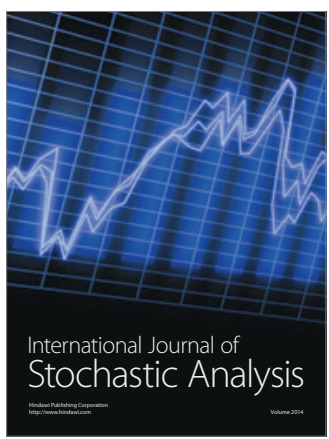

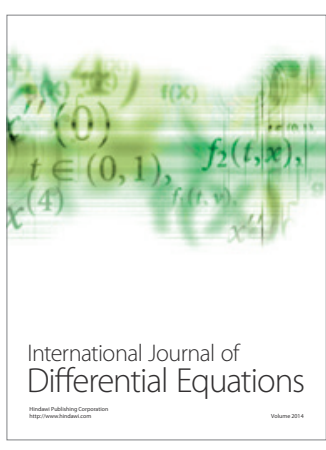
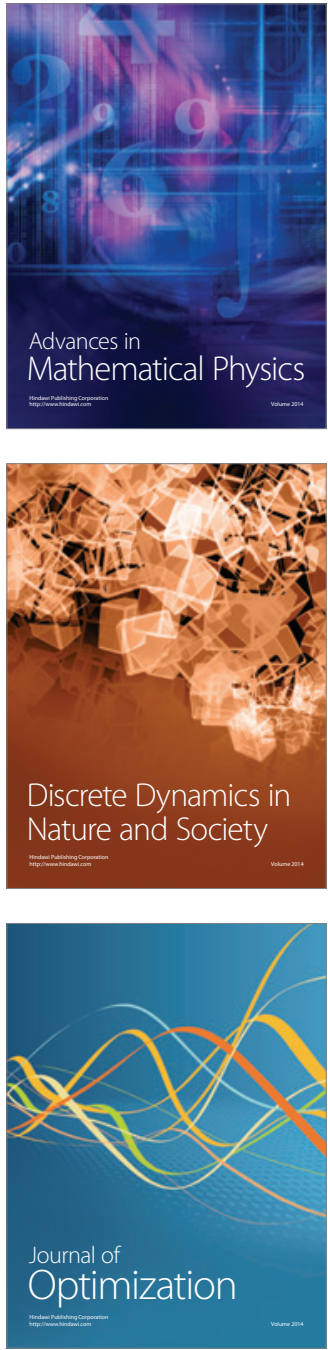